\title{
Diagonally Dominant Backstepping Autopilot for Aircraft with Unknown Actuator Failures and Severe Winds
}

\author{
Shaik Ismail ${ }^{1}$ and Abhay A. Pashilkar ${ }^{2}$ \\ National Aerospace Laboratories, Bangalore-560017, India \\ Ramakalyan Ayyagari ${ }^{3}$ \\ National Institute of Technology, Tiruchirappalli-620015, India \\ and \\ Narasimhan Sundararajan ${ }^{4}$ \\ Nanyang Technological University, Singapore 639798
}

This paper presents a novel formulation of the flight dynamic equations that permits a rapid solution for the design of trajectory following autopilots for nonlinear aircraft dynamic models. A robust autopilot control structure is developed based on the combination of the good features the Nonlinear Dynamic Inversion (NDI) method, Integrator Backstepping method, Time Scale separation and Control Allocation methods. The aircraft equations of motion are formulated in suitable variables so that the matrices involved in the block backstepping control design method are diagonally dominant. This allows us to use a linear controller structure for a trajectory following autopilot for the nonlinear aircraft model using the well known loop by loop controller design approach. The resulting autopilot for the fixed-wing rigidbody aircraft with a cascaded structure is referred to as the Diagonally Dominant Back-Stepping (DDBS) controller. The method is illustrated here for an aircraft auto-landing problem under unknown actuator failures and severe winds. The issue of state and control surface limiting is also addressed in the context of the design of the DDBS controller.

\section{Nomenclature}

A

$b$

B

BBS

$C_{L \alpha}$

CFD

$D$ linear system matrix for rotational dynamics control effectiveness derivative(s)

linear control matrix for rotational states and aerodynamic inputs block backstepping

lift curve slope with angle of attack computational fluid dynamics aircraft drag, $\mathrm{N}$

\footnotetext{
1 Senior Principal Scientist (retd.), Flight Mechanics and Control Division, Post Box No. 1779, shaik1752@gmail.com.

${ }^{2}$ Group Head, Simulation Group, Flight Mechanics and Control Division, Post Box No. 1779, apash@nal.res.in.

${ }^{3}$ Professor, Dept. of Instrumentation and Control Engineering, rkalyn@nitt.edu.

${ }^{4}$ Professor (retd.), School of Electrical and Electronic Engineering, ensundara@ntu.edu.sg.
} 
DDBS

e

$f$

FDIA

$g$

h

I

K

$l$

$V_{\text {lyap }}$

$L$

LHP

$m$

M

MIMO

$n$

NDI

$p$

PID

$q$

qbar

$r$

RHP

SISO

$\mathrm{S}$

S

$\mathrm{T}$

T

u

u

UAV

$v$

V

$w$

$\mathbf{x}$

y

$\mathbf{y}$

$Y$

$\alpha$

$\beta$

$\delta$

$\chi$

$\gamma$

diagonally dominant backstepping

error in the state variable $\left(x-x^{d}\right)$

nonlinear function of state(s)

fault detection, identification and accomodation

acceleration due to gravity, $9.81 \mathrm{~m} / \mathrm{s}^{2}$

aircraft altitude, $\mathrm{m}$

moment of inertia matrix, $\mathrm{kgm}^{2}$

feedback gain

rolling moment, $\mathrm{Nm}$

Lyapunov function

Lift force, $\mathrm{N}$

left half of the complex plane

pitching moment, $\mathrm{Nm}$

mass of aircraft, kg

multi-input-multi-output

yawing moment, $\mathrm{Nm}$

nonlinear dynamic inversion

roll rate, $\mathrm{rad} / \mathrm{sec}$

proportional-integral-derivative

pitch rate, $\mathrm{rad} / \mathrm{sec}$

dynamic pressure, $\mathrm{N} / \mathrm{m}^{2}$

yaw rate, $\mathrm{rad} / \mathrm{sec}$

right half of the complex plane

single-input-single-output

wing area, $\mathrm{m}^{2}$

control ganging matrix

engine thrust, $\mathrm{N}$

rotational transformation matrix

aircraft velocity along body $\mathrm{x}$-axis, $\mathrm{m} / \mathrm{s}$

vector of control inputs $\mathbf{u}=\left[\begin{array}{lllll}\delta_{e-l e f t} & \delta_{e-r i g h t} & \delta_{a-l e f t} & \delta_{a-\text { right }} & \delta_{r}\end{array}\right]^{T}$

uninhabited aerial vehicle

aircraft velocity along body y-axis, $\mathrm{m} / \mathrm{s}$

velocity of aircraft, $\mathrm{m} / \mathrm{s}$

aircraft velocity along body z-axis, $\mathrm{m} / \mathrm{s}$

state vector of rotational states $\mathbf{x}=\left[\begin{array}{lll}q & p & r\end{array}\right]^{T}$

lateral displacement of aircraft towards east, $\mathrm{m}$

vector of aerodynamic states $\mathbf{y}=\left[\begin{array}{lllll}\alpha & q & \beta & p & r\end{array}\right]^{T}$

side force in wind axis, $\mathrm{N}$

angle of attack, deg

angle of sideslip, deg

aerodynamic control surface deflection, deg

ground track angle, deg

flight path angle, deg 
$\eta$
$\phi$
$\theta$
$\psi$
$\mu$
$\tau$

\section{Superscripts}

$\wedge$

\section{Subscripts}

\section{$a$}

aei

alpha

ari

a-left

a-right

beta

cmd

chi

e-left

e-right

gam

$h$

$m u$

pail

pele

pitch

prud

ps

$q$

qele

$r$

rei

rele

roll

rrud

rs

$S$

thr

trim

$\mathrm{T}$

vthr

vel

$y$ positive constant

aircraft bank angle, deg

aircraft pitch angle, deg

aircraft heading angle, deg

angle of roll about velocity vector, deg

time constant, sec

estimate of the parameter

vector transformed to stability axis

aerodynamic contribution

differential elevator due to roll command

angle of attack

aileron to rudder interconnect

left aileron deflection, deg

right aileron deflection, deg

angle of sideslip

command

ground track angle

left elevator deflection, deg

right elevator deflection, deg

flight path angle

altitude

roll angle about velocity vector

roll due to aileron

roll due to differential elevator

pitch axis pseudo control, deg

roll due to rudder

stability axis roll rate

pitch rate

pitch due to elevator

right aileron deflection, deg

differential elevator due to yaw command

yaw due to elevator

roll axis pseudo control, deg

yaw due to rudder

stability axis yaw rate

stability axis

throttle input, range (0-1)

value of parameter at trim

engine contribution

control derivative of rate of change velocity equation w.r.t throttle velocity

lateral deviation along trajectory 


$\begin{array}{ll}\text { yaw } & \text { yaw axis pseudo control, deg } \\ 1 & \text { first element of a vector } \\ 2 & \text { second element of a vector }\end{array}$

\section{Introduction}

Flight control design is a challenging problem because it involves finding the right control inputs given the aircraft dynamics such that its outputs follows a desired trajectory. Flight controller design is a well known inverse design problem in the areas of flight dynamics and control [1].

This problem is compounded by the presence of nonlinearities in the equations of motion as well as the uncertain aerodynamic forces and moments. Further, this nonlinear dependence of the aerodynamic forces and moments is not known exactly and the controller must be robust against reasonable variations in the plant parameters. All the traditional linear Single-Input-SingleOutput (SISO) and Multi-Input-Multi-Output (MIMO) design techniques have been used in aircraft flight controller design. Many of these techniques make use of some aspect of the nonlinear dynamic inversion principle while choosing the variables for feedback and the controller structure.

Block Backstepping (BBS) is a well known technique for the design of nonlinear controllers [27] based on nonlinear dynamic inversion principle. In this paper, we show that by a suitable choice of variables in the aircraft dynamic equations, the 3x3 matrices involved in the inversion process to generate a BBS controller are rendered diagonally dominant and hence can be designed using linear controller design methods. The resulting controller which we call a Diagonally Dominant Backstepping (DDBS) controller has a cascaded linear controller structure for the class of fixed wing aircraft described by the nonlinear six-degree-of-freedom equations. Essentially, it means that we can do loop by loop design of the full trajectory following controller for a nonlinear aircraft using a linear controller structure by the proposed approach.

Concepts from the design methods such as Nonlinear Dynamic Inversion (NDI), Integrator Backstepping, Time Scale Separation and Control Allocation are combined here to produce a robust controller design. Furthermore, the controller so derived is linear permitting us to provide the gain and phase margins required for certification. This approach can be used to develop quickly a trajectory following autopilot for any aircraft without going through a tedious and time consuming approach.

Figure 1 presents the overall structure of the proposed DDBS controller and the state variables involved at each stage of the dynamic inversion process. A static control allocation block is provided which improves the failure tolerance, explained in section III.B. The cascaded controller has control loops which follow the block triangular structure of the aircraft equations of motions, viz inner loop control (i.e., rotational equations), wind axis control (wind axis equations), trajectory control (i.e., velocity vector equations) and position control (i.e., navigation equations). It is seen that the inner most loop represents the fastest dynamics (highest 
bandwidth) and the intermediate loops have progressively decreasing bandwidth with the outer most loop having the slowest dynamic variables. Before describing our approach, a brief review of exisiting aircraft control methods is given below.

\section{A. Review of Nonlinear Inversion and Backstepping Design Methods for Flight Control}

The most widely studied approach to nonlinear flight controller design is based on nonlinear transformation techniques, commonly known as feedback linearization [8]. This methodology transforms a nonlinear system into a system exhibiting linear dynamics so that linear control methods can be applied to it. The earliest application of feedback linearization in flight control research is described in [9]. In a later work, a time-scale approach was used to simplify the linearizing transformations [10]. A specific case of feedback linearizing control, known as NDI, has been investigated extensively for application to control of super-maneuverable aircraft [1113] culminating in flight testing of NDI based control laws [14]. The requirement for supermaneuverability also requires that the control system protect the aircraft from entering regimes of flight which can result in a loss of control. In [11] this problem has been solved by switching between alternate command variable sets depending on the phase of flight.

Although NDI is an effective way of compensating for nonlinearities associated with high angle-of-attack flight, it is very vulnerable to modeling errors [15]. Therefore, a variety of robust nonlinear control schemes that can be used in conjunction with the NDI methodology have been proposed. These techniques provide robustness against modeling and parametric uncertainties, and uncertain nonlinearities [15-17].

Another approach to control a nonlinear system that does not rely entirely on dynamic inversion is the class of so-called backstepping techniques [2]. Backstepping uses a recursive synthesis procedure to determine the nonlinear controller for linear or nonlinear systems with a particular cascaded structure. The block triangular nature of the aircraft equations of motion allows us to apply backstepping for flight controller design. Backstepping improves the robustness of NDI significantly by introducing a feedback element into the control structure. Due to this positive attribute, backstepping was applied to the problem of flight control $[3-7,18]$. In these works, we find a clear demarcation of the states involved in the block triangular form of the equations of motion along with the explicit intent to design the flight control system based on multiple time scale separation principles.

As shown in [3, 5-6], it is not necessary to cancel all nonlinearities in the dynamic equations of motion. In fact, in many cases it is possible to retain the useful nonlinearities and thereby reduce the control effort. Further, in many cases a sufficiently high linear negative feedback gain can make the adaptive backstepping controller globally asymptotically stable. In [4] these results have also been extended to non-affine systems.

A comparison of NDI, backstepping and related cascade design methods for nonlinear flight controller design is given in [5] and [6]. The resulting control laws specify the total control effort to produce, but not on how to produce it (mainly the allocation to different control surfaces). Modern high performance aircraft are typically over-actuated for ensuring adequate safety and performance. There are several combinations of control surface deflections that will give the 
same aircraft response. In [19] it is shown how multiple redundant control surfaces could be handled in the NDI framework using a matrix pseudo-inverse. The various methods of control allocation available to the control law designer - namely explicit ganging, pseudo control, pseudo inverse, and daisy chaining are discussed here. Further, the process of designing feed forward loops to improve the tracking performance of the dynamic inversion controller is also highlighted here.

Use of stability axis rates as opposed to body axis rates in the innermost loops as a natural choice for flight controller design is clearly seen in [15] and [19].

Trajectory following controller designs based on adaptive online function approximation has also been developed in [7, 20]. These methods use integrator backstepping and function approximation methods which are linear in parameters. Stability has been proved in the sense of Lyapunov.

It is noted that while NDI in combination with backstepping have been successfully applied to many fixed wing aircraft $[3-7,18,20]$, the resulting nonlinear controllers suffer from the following deficiencies:

a. It has been shown in [3, 5-6] that attempting to cancel every nonlinear term is not the best approach and better transient performance can be obtained by a suitable choice of the linear controller. We show in this paper that by reformulating the right hand side of the equations of motion using a particular combination of variables, the diagonally dominant nature of the block backstepping becomes apparent. This allows us to derive a linear cascaded controller structure for a trajectory following autopilot for a nonlinear aircraft and the loop by loop design is possible.

b. Airworthiness authorities require guarantees of stability for the autopilots for fixed wing aircraft and UAV's for safe operation. The guarantee of stability for NDI based design approaches is provided by Lyapunov methods. There is no unique Lyapunov function which is applicable to the aircraft control problem and each researcher chooses his/her own. The conditions on the controller gains can be conservative due to the non-uniqueness of the Lyapunov function. The computation of the domain of attraction for the nonlinear controller is difficult in multi-dimensional space. This is in contrast to the linear design techniques which provide robustness guarantees through gain and phase margins which are accepted by certification authorities.

c. Adaptive control solutions [7, 20] have been proposed for fault tolerant control. It is clear that online estimation of the aircraft dynamics improves the performance of the controller if sufficient time is given for the parameter values to converge. Therefore, even an adaptive controller must possess sufficiently high gains to guarantee stability during this finite time interval required for learning the changed dynamics. This is also true for controllers which use the principle of Fault Detection, Isolation and Accomodation (FDIA) and need this finite time interval to effect a reconfiguration.

d. Lyapunov based methods available in literature do not provide a systematic way of arriving at the controller gains. Autopilot designs for longitudinally unstable aircrafts must ensure that the very high outer loop gains do not saturate the actuators resulting in a loss of control. This is especially true when the aircraft has to survive severe unknown winds such as those used in this paper. The time scale separation principle becomes particularly important while choosing the gains in such cases. 
e. As pointed out in [21], it is important to consider all the hardware elements and their effect on the closed loop stability and performance In the literature, we find that while many researchers have accounted for actuator position and rate limits, the computational delays inherent in the digital implementation of the control laws have not been accounted for. This delay adds to the overall lag in the system and thus limits the maximum gain that can be used in the innermost loop. In our work we consistently use a lumped computational lag of 40msec (twice the sampling interval) representing the input as well as output delays.

The aim of the present work is to use the NDI and integrator backstepping concepts to develop a linear controller that can be used to rapidly design a baseline autopilot for the nonlinear aircraft dynamics. The choice of the linear controller is motivated by the fact that sufficiently high linear gains can make the system globally asymptotically stable even if we do not cancel some of the nonlinearities [3, 5-6]. The approach presented in this paper allows us to exploit linear analysis tools which are well developed and the traditional gain and phase margins can be easily provided for certification [19]. A novel feedback controller scheme is proposed in a cascaded form in this paper and that addresses both the state variable limiting and control surface saturation specifications.

We use wind axis coordinates, particularly the wind axis roll and yaw rates, to simplify the controller sufficiently, permitting us to neglect the nonlinear terms while retaining stability. We also demonstrate how one can select the gains in the various loops whilst maintaining dynamic time scale separation between the inner and outer loops. This approach is sufficiently generic to allow for application to many class of fixed wing aircraft, including Unmanned Air Vehicles (UAVs).

The use of integrator backstepping allows us to change the loop bandwidth directly preventing control surface position / rate saturations [12-13]. A simple and fixed control allocation scheme is also described in this paper that increases the robustness of the controller for unknown actuator failures and also it simultaneously decouples the lateral and directional equations of motion.

The rest of the paper is organized as follows. Section II describes the aircraft model and the actuator models used. The linear model data which forms the basis for innermost loop design is also provided in this Section. The basic ideas behind NDI and backstepping are described. In Section III we present the conventional model formulation used to design a BBS controller. This is followed by the DDBS controller design proposed in this paper. The DDBS equations of motion result in a loop by loop cascade design structure which is similar to that used in classical SISO design approach. In Section IV, we first present a comparison between the two autopilot designs, one based on the classical SISO approach and the other based on DDBS linear controller. The six-degree-of-freedom (6 DOF) simulations are presented in Section V. Conclusions from this study are summarized in Section VI. 


\section{Aircraft Model and Conventional Block Backstepping Approach}

\section{A. Aircraft Dynamics}

The aircraft model used in this study is that of a high performance fighter aircraft [22]. For the present study, the elevator and aileron control surface aerodynamics data have been split into two parts corresponding to the left and right surfaces using CFD computations [23]. The independent left and right elevators can be moved together or in differential mode, and the deflection range is -25 to +25 deg. Similarly, the ailerons can be deflected independently from -20 to +20 deg., and the rudder from -30 to $+30 \mathrm{deg}$. The aerodynamic model also contains a ground effect model. The engine model with a 5 sec time constant simulates the overall lag of the power plant.

The aircraft has hydraulic actuators for deflecting the primary control surfaces, and are modeled as first order lags with a time delay of $50 \mathrm{~ms}$. The rate limit for the actuators is set at 60 $\operatorname{deg} / \mathrm{s}$.

\section{B. Linear Aircraft Model}

The design of a prototype nonlinear controller based on NDI, integrator backstepping, and control allocation concepts is described in this Section. A linear model of the high performance aircraft is required for designing the inner-most control loop dealing with the fast rotational states. The linear model required for the design of the inner-most loop can be expressed in statespace form as:

$$
\dot{\mathbf{x}}=\mathbf{A y}+\mathbf{B u}
$$

where, the vectors $\mathbf{x}, \mathbf{y}$ and $\mathbf{u}$ are defined as:

$$
\begin{aligned}
& \mathbf{x}=\left[\begin{array}{lll}
q & p & r
\end{array}\right]^{T} \\
& \mathbf{y}=\left[\begin{array}{lllll}
\alpha & q & \beta & p & r
\end{array}\right]^{T} \\
& \mathbf{u}=\left[\begin{array}{lllll}
\delta_{e-l e f t} & \delta_{e-r i g h t} & \delta_{a-l e f t} & \delta_{a-r i g h t} & \delta_{r}
\end{array}\right]^{T}
\end{aligned}
$$

Where in (1), $\mathbf{A}$ and $\mathbf{B}$ are the plant and control matrices respectively. It should be noted that (1) is different from the traditional form of the linear model equations because we have explicitly introduced here the primary aerodynamic dependency on the angle of attack and sideslip into the equations in addition to the dependence on the angular rates.This linear model of the aircraft was obtained by trimming the nonlinear model at the straight and level flight condition $(V=82.66$ $\mathrm{m} / \mathrm{s}, h=600 \mathrm{~m})$. The equilibrium values of the sate variables are: $u=81.31 \mathrm{~m} / \mathrm{s}, v=0 \mathrm{~m} / \mathrm{s}, w=$ $16.08 \mathrm{~m} / \mathrm{s}, p=0 \mathrm{rad} / \mathrm{s}, q=0 \mathrm{rad} / \mathrm{s}, r=0 \mathrm{rad} / \mathrm{s}, \phi=0 \mathrm{rad}, \theta=0.1853 \mathrm{rad}, \psi=0 \mathrm{rad}, h=600 \mathrm{~m}$, and $y=0 \mathrm{~m}$. Similarly, the trim values of control deflections are: $\delta_{e-l e f t}=\delta_{e-r i g h t}=-0.64 \mathrm{deg}$, $\delta_{a-l e f t}=\delta_{a-r i g h t}=0 \mathrm{deg}, \delta_{r}=0 \mathrm{deg}$, and $\delta_{t h r}=0.22$. It is to be noted that the angles and rates in the state equations are in radians and $\mathrm{rad} / \mathrm{s}$, while the control surface deflections are in degrees.

The numerical values of the matrices $A$ and $B$ are as follows:

$$
\mathbf{A}=\left[\begin{array}{lrrrr}
0.8145 & -0.6491 & -0.0066 & 0 & -0.0029 \\
0 & 0.0003 & -14.1548 & -1.7533 & 0.8792 \\
0 & 0.0025 & 1.8847 & -0.0482 & -0.2424
\end{array}\right]
$$




$$
\mathbf{B}=\left[\begin{array}{rrrrr}
-0.0299 & -0.0299 & 0.0005 & 0.0005 & 0 \\
0.0549 & -0.0549 & 0.0842 & -0.0842 & 0.0340 \\
0.0074 & -0.0074 & 0.0007 & -0.0007 & -0.0169
\end{array}\right]
$$

It is important to note that the structure of the control matrix is as follows:

$$
\mathbf{B}=\left[\begin{array}{ccccc}
-b_{\text {qele }} & -b_{\text {qele }} & 0 & 0 & 0 \\
b_{\text {pele }} & -b_{\text {pele }} & b_{\text {pail }} & -b_{\text {pail }} & b_{\text {prud }} \\
b_{\text {rele }} & -b_{\text {rele }} & 0 & 0 & -b_{\text {rrud }}
\end{array}\right]
$$

It is seen that as expected for a conventional arrangement of control surfaces, the pitch, roll and yaw axes are primarily affected by the elevator, aileron and rudder respectively for this aircraft. The control coupling in the roll axis of this aircraft is seen in terms of the roll due to elevator and rudder. Yaw control coupling from differential elevator is also seen to a smaller extent.

\section{NDI and Backstepping with Linear Feedback}

The basic ideas behind using linear feedback while adopting NDI and backstepping control principles will be illustrated with an affine system with two scalar state variables $\left(x_{1}, x_{2}\right)$ and one control input $\delta$ :

$$
\begin{aligned}
& \dot{x}_{1}=f_{1}\left(x_{1}, x_{2}\right)+b_{1} \delta \\
& \dot{x}_{2}=f_{2}\left(x_{2}\right)+b_{2} x_{1}
\end{aligned}
$$

In (8), $b_{1}, b_{2}$ are scalars and $f_{1}, f_{2}$ are smooth functions of their arguments. A conventional dynamic inversion approach will require us to invert the first of the two equations in (8) to obtain $\delta$ as a function of the state derivative $\dot{x}_{1}$ and $f_{1}$. Similarly, the second equation in (8) is inverted to give $x_{1}$ as a function of the state derivative $\dot{x}_{2}$ and $f_{2}$. In these two inverse functions we then substitute the derivatives of the desired trajectory to obtain:

$$
\begin{aligned}
\delta & =b_{1}^{-1}\left(\dot{x}_{1}^{d}-f_{1}\left(x_{1}, x_{2}\right)\right) \\
x_{1}^{d} & =b_{2}^{-1}\left(\dot{x}_{2}^{d}-f_{2}\left(x_{2}\right)\right)
\end{aligned}
$$

Integrator backstepping is already inherent in the above equation, because the derivative of the desired trajectory $x_{1}^{d}$ obtained by inversion of the second equation in (8) is used in the first equation in (9) to compute the control signal $\delta$. Therefore, the state $x_{1}$ is used as a pseudocontrol. It is noted that for such a control law to exist both the scalars $b_{1}, b_{2}$ must not change sign and the desired trajectory $\left(x_{1}^{d}, x_{2}^{d}\right)$ must lie within the domain bounded by any physical constraints on the state variables. The scalar $b_{1}$ is the derivative of $\dot{x}_{1}$ with respect to the control input $u$ frequently called control derivative in aircraft stability and control literature. On the other hand, $b_{2}$ is the derivative of $\dot{x}_{2}$ with respect to the pseudo-control $x_{1}$. Further, tracking the desired trajectory requires perfect knowledge of the plant dynamics. The other aspect of these control laws is that they operate on the derivatives of the desired trajectory. This means that if there is an initial condition error or external disturbances along the trajectory, there is no inherent 
mechanism to drive the error $\left(e_{1}=x_{1}-x_{1}^{d}, e_{2}=x_{2}-x_{2}^{d}\right)$ to zero. We propose to solve these lacunae by modifying the control laws in (9) as follows:

$$
\begin{aligned}
\delta & =\hat{b}_{1}^{-1}\left(\dot{x}_{1}^{d}-k_{1} e_{1}-\hat{f}_{1}\left(x_{1}, x_{2}\right)\right) \\
x_{1}^{d} & =\hat{b}_{2}^{-1}\left(\dot{x}_{2}^{d}-k_{2} e_{2}-\hat{f}_{2}\left(x_{2}\right)\right)
\end{aligned}
$$

It is noted that terms proportional and opposite to the trajectory error have been added in the two equations multiplied by positive constants $k_{1}, k_{2}$. Also, in order to distinguish between the actual values of the scalars $b_{1}, b_{2}$ and the functions $f_{1}, f_{2}$ as compared to their estimates, they are indicated by the superscript ' $\wedge$ '. The estimated values of these parameters are typically obtained from wind tunnel test data, computational fluid dynamic calculations or empirical methods. Our claim is that with this modification, provided sufficiently large positive scalar gains $k_{1}, k_{2}$ can be found the trajectory errors converge to zero. Consider the Lyapunov function:

$$
V_{\text {lyap }}=e_{1}^{2} / 2+e_{2}^{2} / 2
$$

Taking the time derivative of the Lyapunov function along the trajectories of the closed loop system gives:

$$
\begin{aligned}
\dot{V}_{\text {lyap }} & =e_{1} \cdot \dot{e}_{1}+e_{2} \cdot \dot{e}_{2} \\
& =\left(f_{1}\left(x_{1}, x_{2}\right)+b_{1} \delta-\dot{x}_{1}^{d}\right) e_{1}+\left(f_{2}\left(x_{2}\right)+b_{2} x_{1}-\dot{x}_{2}^{d}\right) e_{2}
\end{aligned}
$$

We now make the assumption that our estimates of the scalars $\hat{b}_{1}, \hat{b}_{2}$ are very close to the actual values in order to simplify the proof. Thus:

$$
b_{1} \hat{b}_{1}^{-1} \cong 1, b_{2} \hat{b}_{2}^{-1} \cong 1
$$

Later we shall show that this assumption is not unduly restrictive. Then substituting the control law given by (10) into (12) gives us:

$$
\dot{V}_{\text {lyap }}=\left(f_{1}\left(x_{1}, x_{2}\right)-\hat{f}_{1}\left(x_{1}, x_{2}\right)-k_{1} e_{1}\right) e_{1}+\left(f_{2}\left(x_{2}\right)-\hat{f}_{2}\left(x_{2}\right)+b_{2} e_{1}-k_{2} e_{2}\right) e_{2}
$$

If the gains $k_{1}, k_{2}$ are chosen sufficiently large so that the following conditions hold:

$$
\begin{aligned}
k_{1} & =\frac{\left|f_{1}\left(x_{1}, x_{2}\right)-\hat{f}_{1}\left(x_{1}, x_{2}\right)\right|}{e_{1}}+\eta_{1} \\
k_{2} & =\frac{\left|f_{2}\left(x_{2}\right)-\hat{f}_{2}\left(x_{2}\right)\right|}{e_{2}}+\eta_{2} \\
\sqrt{\eta_{1} \eta_{2}} & >\left|b_{2}\right|
\end{aligned}
$$

We can express the equality in (14) as the inequality:

$$
\dot{V}_{\text {lyap }} \leq-\left(\sqrt{\eta_{1}} e_{1}-\sqrt{\eta_{2}} e_{2}\right)^{2}
$$

Where $\eta_{1}, \eta_{2}$ are positive scalars. This proves the convergence of the errors to zero in the sense of Lyapunov provided sufficiently large gains can be found to dominate the errors in the function estimates $\left|f_{1}\left(x_{1}, x_{2}\right)-\hat{f}_{1}\left(x_{1}, x_{2}\right)\right|,\left|f_{2}\left(x_{2}\right)-\hat{f}_{2}\left(x_{2}\right)\right|$. The conditions (15) imply that the gains $k_{1}, k_{2}$ are large enough so the in case the gradient of the functions $f_{1}, f_{2}$ with respect to the state variables are destabilizing, the net resultant gradient becomes stabilizing. This means that even for online adaptive controllers, sufficiently high gains are required to maintain stability during the online learning phase. The above proof considers a system of two states $x_{1}, x_{2}$. In case of 
trajectory following for fixed wing aircraft, up to four sets of cascaded equations are involved. We can extend the result for a system of equations in the block triangular form with more than two states by suitable conditions on the gains [24].

Consider the case where $\hat{f}_{1}=\hat{f}_{2}=0$, which means that we do not cancel the nonlinearities inherent in the equations of motion. If the functions $f_{1}, f_{2}$ have a negative gradient with respect to $x_{1}, x_{2}$ respectively, then these nonlinearities are inherently stabilizing and we need not cancel them to ensure stability as seen in (14). In the event the functions $f_{1}, f_{2}$ are indeed destabilizing, stability of the closed loop control system can be ensured even if we do not cancel the nonlinearities provided we find sufficiently high values for the gains $k_{1}, k_{2}$ to satisfy (15) with $\hat{f}_{1}=\hat{f}_{2}=0$. The condition on the control derivatives $\hat{b}_{1}, \hat{b}_{2}$ in (13) can also be relaxed if the control gains are high enough to dominate both the approximation errors as well as the nonlinearities. In fact, we show in the next sub-section that except for the inner most loop $\left(p_{s}, q, r_{s}\right)$, the flight path angle $(\gamma)$ and velocity $(V)$ loops, the control derivatives for all other control loops do not contain any aircraft specific parameters and are therefore known exactly. Therefore in conclusion, if sufficiently large linear gains are found it is possible to develop a robust linear design for a block triangular system of equations such as that found in the case of fixed wing aircraft. This is the main result used in this paper to demonstrate the application of this to a trajectory following autopilot in the subsequent sections.

It may be noted that although a Lyapunov based proof has been used to motivate the design of the linear autopilot, the resulting design allows for the computation of loop by loop gain and phase margins using linear aircraft models. The final point to note is that in (10), the control laws require computation of the derivatives of the desired states, namely $\dot{x}_{1}^{d}, \dot{x}_{2}^{d}$. One approach used to compute these derivatives is to implement a second order command filter with position and rate limits incorporated into the filter $[7,18,20]$. This approach gives better stability properties and performance for the closed loop [6].

The other approach to implement control laws in (10) is to choose the gain $k_{1}$ to maintain sufficient time scale separation between actuator dynamics $(\delta)$ and the state $x_{1}^{d}[10,5-6,24]$. Similarly, we maintain sufficient separation between the states $x_{1}^{d}$ and $x_{2}^{d}$ by suitable choice of $k_{2}$. This means that the actuator dynamics is fast enough compared to $x_{1}^{d}$, so that we can assume $\dot{x}_{1}^{d}=0$ while computing $\delta$ in (10). In a similar fashion we can assume $\dot{x}_{2}^{d}=0$ while computing $x_{1}^{d}$ for the control law in (10). In this paper we will first construct the feedback gains assuming time scale separation. Subsequently, we will design washout filters based on the time scale of each loop to compute the derivatives required to implement (10).

\section{Proposed Formulation and Diagonally Dominant Backstepping Controller Design}

In the previous section, we have demonstrated the benefits of combining the ideas of nonlinear dynamic inversion as well as backstepping for the control of flight vehicles. In this section we show how BBS is simplified into DDBS. In DDBS we achieve a loop-by-loop design 
process which is rapid and allows us to retain all the benefits of NDI and backstepping. The key to this lies in formulating the equations of motion in a mixed axis system of variables.

\section{A. Block Backstepping Controller Design}

The six-degree-of-freedom equations of motion of rigid body fixed-wing aircraft are given in four groups of three first order nonlinear differential equations. For details, see [7]. In the BBS approach, the control / pseudo control signal is constructed by simultaneously computing the dynamic inverse of the set of three equations at a time. In this section, we elaborate on this process for the design of the BBS control law. These groups of equations are already depicted schematically in Fig. 1.

Rotational Equations:

The rotational equations of motion are given in the body-axis system:

$$
\left[\begin{array}{c}
\dot{p} \\
\dot{q} \\
\dot{r}
\end{array}\right]=\mathbf{I}^{-1}\left\{-\left[\begin{array}{ccc}
0 & -r & q \\
r & 0 & -p \\
-q & p & 0
\end{array}\right] \mathbf{I}\left[\begin{array}{l}
p \\
q \\
r
\end{array}\right]+\left[\begin{array}{l}
l_{a} \\
m_{a} \\
n_{a}
\end{array}\right]+\left[\begin{array}{l}
l_{T} \\
m_{T} \\
n_{T}
\end{array}\right]\right\}
$$

The inner loop (Fig. 1) of the BBS is constructed by computing the dynamic inverse of (17) to give the three aerodynamic moments $\left(l_{a}, m_{a}, n_{a}\right)$ as a function of the commanded angular rate derivatives $\left(\dot{p}_{c m d}, \dot{q}_{c m d}, \dot{r}_{c m d}\right)$. In (17), $\left(l_{T}, m_{T}, n_{T}\right)$ are the moments due to engine thrust. In addition, backstepping introduces a feedback signal proportional to the error between commanded and actual angular rates $\left(p_{c m d}-p, q_{c m d}-q, r_{c m d}-r\right)$. As explained in the Section II.C, this feedback is crucial for stability and performance of the controller. The control allocation block is used to apportion the computed aerodynamic moments to individual control surface deflections. In the following section on the DDBS controller, we demonstrate that it is more appropriate to work with the stability axis angular rates $\left(p_{s}, q, r_{s}\right)$ rather than body axis rates $(p, q, r)$. Also, we demonstrate how to simultaneously achieve control decoupling in the stability axis.

Wind Axis Equations:

$$
\left[\begin{array}{c}
\dot{\mu} \\
\dot{\alpha} \\
\dot{\beta}
\end{array}\right]=\left[\begin{array}{ccc}
\frac{\operatorname{Cos} \alpha}{\operatorname{Cos} \beta} & 0 & \frac{\operatorname{Sin} \alpha}{\operatorname{Cos} \beta} \\
-\operatorname{Cos} \alpha \operatorname{Tan} \beta & 1 & -\operatorname{Sin} \alpha \operatorname{Tan} \beta \\
\operatorname{Sin} \alpha & 0 & -\operatorname{Cos} \alpha
\end{array}\right]\left[\begin{array}{c}
p \\
q \\
r
\end{array}\right]+\left[\begin{array}{cc}
\operatorname{Sin} \gamma+\operatorname{Cos} \gamma \operatorname{Sin} \mu \operatorname{Tan} \beta & \operatorname{Cos} \mu \operatorname{Tan} \beta \\
-\frac{\operatorname{Cos} \gamma \operatorname{Sin} \mu}{\operatorname{Cos} \beta} & -\frac{\operatorname{Cos} \mu}{\operatorname{Cos} \beta} \\
\operatorname{Cos} \gamma \operatorname{Cos} \mu & -\operatorname{Sin} \mu
\end{array}\right]\left[\begin{array}{c}
\dot{\chi} \\
\dot{\gamma}
\end{array}\right]
$$

The above formulation is found in [7]. The wind axis control loop (Fig. 1) of the BBS is constructed by computing the dynamic inverse of (18) to give the angular rate commands $\left(p_{c m d}, q_{c m d}, r_{c m d}\right)$ as a function of the commanded wind axis angle rates $\left(\dot{\mu}_{c m d}, \dot{\alpha}_{c m d}, \dot{\beta}_{c m d}\right)$. The first $3 \mathrm{x} 3$ matrix on the right hand side of (18) is required to be inverted in this process. We show in the section on DDBS how the choices of stability axis roll and yaw rates considerably simplify 
the inversion. Also the role of the gravity terms which is not apparent in the second term in (18) is revealed explicitly by suitable choice of variables.

Velocity Vector Equations:

$$
\left[\begin{array}{c}
\dot{V} \\
\dot{\chi} \\
\dot{\gamma}
\end{array}\right]=\left[\begin{array}{c}
\frac{(T \operatorname{Cos} \alpha \operatorname{Cos} \beta-D)}{M}-g \operatorname{Sin} \gamma \\
\frac{L \operatorname{Sin} \mu}{M V \operatorname{Cos} \gamma}+\frac{T(\operatorname{Sin} \alpha \operatorname{Sin} \mu-\operatorname{Cos} \alpha \operatorname{Sin} \beta \operatorname{Cos} \mu)}{M V \operatorname{Cos} \gamma}+\frac{Y \operatorname{Cos} \mu}{M V \operatorname{Cos} \gamma} \\
\frac{T(\operatorname{Sin} \alpha \operatorname{Cos} \mu+\operatorname{Cos} \alpha \operatorname{Sin} \beta \operatorname{Sin} \mu)}{M V}-\frac{Y \operatorname{Sin} \mu}{M V}+\frac{(L \operatorname{Cos} \mu-M g \operatorname{Cos} \gamma)}{M V}
\end{array}\right]
$$

The trajectory control loop (Fig. 1) of the BBS is constructed by inverting (19) to determine the commands $\left(\mu_{c m d}, \alpha_{c m d}, \delta_{t h r}\right)$ given values of the commanded rates $\left(\dot{\chi}_{c m d}, \dot{\gamma}_{c m d}, \dot{V}_{r e f}\right)$. The above equations contain terms with lift, drag, sideforce and thrust. There are also terms with gravity included. However significant simplification of (19) for purposes of control design is achieved if we use the fact that for a fixed wing aircraft, the lift vector is the primary source for changing the curvature of the flight path in both directions (i.e., to create $\dot{\chi}, \dot{\gamma}$ ). This has not been emphasized in the BBS design literature [2-7]. We show this in the following section on the DDBS controller.

Navigational Equations:

$$
\left[\begin{array}{c}
\dot{x} \\
\dot{y} \\
\dot{h}
\end{array}\right]=V\left[\begin{array}{c}
\operatorname{Cos} \gamma \operatorname{Cos} \chi \\
\operatorname{Cos} \gamma \operatorname{Sin} \chi \\
\operatorname{Sin} \gamma
\end{array}\right]
$$

The navigation equations above are simple. They are commonly used in BBS controllers and we shall retain them without change for the DDBS controller. The deviation of the aircraft from the desired altitude and ground track is used to create pseudo commands in the flight path variables $\left(\gamma_{c m d}, \chi_{c m d}\right)$.

\section{B. Diagonally Dominant Backstepping Controller Design}

A mixed-axis system is used for simplifying the design of control laws based on dynamic inversion.

\section{Rotational Equations:}

The rotational equations are given in (17). We first transform to the stability axes:

$$
\left[\begin{array}{c}
p_{s} \\
r_{s}
\end{array}\right]=\left[\begin{array}{cc}
\operatorname{Cos} \alpha & \operatorname{Sin} \alpha \\
-\operatorname{Sin} \alpha & \operatorname{Cos} \alpha
\end{array}\right]\left[\begin{array}{l}
p \\
r
\end{array}\right]=\mathbf{T}_{s}\left[\begin{array}{l}
p \\
r
\end{array}\right]
$$

Where, $\alpha$ is the angle of attack, and $\mathbf{T}_{s}$ is the body-axis to stability-axis transformation matrix.

Consider the three pseudo-controls:

$$
\overline{\mathbf{u}}=\left[\begin{array}{lll}
\delta_{\text {pitch }} & \delta_{\text {roll }} & \delta_{\text {yaw }}
\end{array}\right]^{T}
$$


These controls are used to exercise decoupled control of each of the three rotational axes. The structure of the control effectiveness matrix in (7) suggests the following approach for control decoupling:

a. The demand by roll channel to ailerons for generating roll axis accelerations is used to proportionally deflect the rudder to create sufficient yaw acceleration to ensure roll about the velocity vector. This is same as the aileron to rudder interconnect gain $K_{a r i}$ used routinely in flight control to ensure that stability axis yaw rate (and therefore sideslip) is suppressed due to roll command.

b. The demand by the yaw channel to rudder for generating yaw axis accelerations is applied proportionally via gain $K_{r e i}$ to differentially deflect the elevators to suppress the resulting roll disturbance. We could also have used the ailerons for this purpose, but choose to use the elevators in this manner as they have a larger deflection range.

c. The demand by the roll channel to ailerons is also applied proportionally to deferentially deflect the elevators via gain $K_{a e i}$ to augment the roll accelerations.

$$
\mathbf{u}=\left[\begin{array}{ccc}
1 & -K_{a e i} & -K_{r e i} \\
1 & K_{a e i} & K_{r e i} \\
0 & -1 & 0 \\
0 & 1 & 0 \\
0 & K_{a r i} & 1
\end{array}\right] \overline{\mathbf{u}}=\mathbf{S} \overline{\mathbf{u}}
$$

The structure of the control matrix given in (7) permits us to fully decouple the controls along pitch, roll and yaw axes whist enhancing the ability to handle multiple failures as we shall show in the discussion below.

We also transform the roll and yaw rates into the stability axis rates. The transformed state and output vectors are given by:

$$
\begin{gathered}
\overline{\mathbf{x}}=\left[\begin{array}{lll}
q & p_{s} & r_{s}
\end{array}\right]^{T} \\
\overline{\mathbf{y}}=\left[\begin{array}{lllll}
\alpha & q & \beta & p_{s} & r_{s}
\end{array}\right]^{T}
\end{gathered}
$$

The matrices which transform the original variables $\mathbf{x}, \mathbf{y}$ and $\mathbf{u}$ to their transformed quantities $\overline{\mathbf{x}}, \overline{\mathbf{y}}$ and $\overline{\mathbf{u}}$ respectively are given by:

$$
\begin{array}{r}
\mathbf{x}=\left[\begin{array}{c|c}
1 & 0_{1 \times 2} \\
\hline 0_{2 \times 1} & \mathbf{T}_{s}^{-1}
\end{array}\right] \overline{\mathbf{x}}=\mathbf{T}_{1} \overline{\mathbf{x}} \\
\mathbf{y}=\left[\begin{array}{c|c}
\mathbf{I}_{3 \times 3} & 0_{3 \times 2} \\
\hline 0_{2 \times 3} & \mathbf{T}_{s}^{-1}
\end{array}\right] \overline{\mathbf{y}}=\mathbf{T}_{2} \overline{\mathbf{y}}
\end{array}
$$

Using the above transformations, the linearized equations for rotational dynamics (1) can be written as:

$$
\mathbf{T}_{1} \dot{\overline{\mathbf{x}}}=\mathbf{A} \mathbf{T}_{2} \overline{\mathbf{y}}+\mathbf{B S} \overline{\mathbf{u}}
$$

The product BS works out to be: 


$$
\mathbf{B S}=\left[\begin{array}{ccc}
-2 b_{\text {qele }} & 0 & 0 \\
0 & -2 b_{\text {pail }}+b_{\text {prud }} K_{\text {ari }}-2 b_{\text {pele }} K_{\text {aei }} & -2 b_{\text {pele }} K_{\text {rei }}+b_{\text {prud }} \\
0 & -b_{\text {rrud }} K_{\text {ari }}-2 b_{\text {rele }} K_{\text {aei }} & -b_{\text {rrud }}
\end{array}\right]
$$

As described in the discussion above, it is noted that the gain $K_{r e i}$ is chosen so that we have zero roll moments induced due to yaw demand. This implies:

$$
-2 b_{\text {pele }} K_{\text {rei }}+b_{\text {prud }}=0
$$

The above condition ensures that $\mathbf{B S}$ is diagonally dominant. In the second column of this matrix product, the gain $K_{a r i}$ is chosen to ensure roll about velocity vector. Thus, control decoupling of the roll and yaw channels is achieved by the two interconnect gains $K_{r e i}$ and $K_{a r i}$. Finally, the gain $K_{a e i}$ is actually optional and chosen to ensure additional fault tolerance capability in terms of being able to roll by differential application of elevators even when both ailerons have failed. In addition to the diagonal dominance of $\mathbf{B S}$, if the diagonal entries do not change sign in the state space (i.e., there is no control reversal) then its inverse always exists.

Accordingly (28) can be rearranged as:

$$
(\mathbf{B S})^{-1} \mathbf{T}_{1} \dot{\overline{\mathbf{x}}}=(\mathbf{B S})^{-1} \mathbf{A} \mathbf{T}_{2} \overline{\mathbf{y}}+\overline{\mathbf{u}}
$$

Where, $(\mathbf{B S})^{-1}$ is the inverse of the matrix product BS. The gains $K_{a r i}, K_{a e i}$ and $K_{r e i}$ in the matrix $S$ are chosen such that $(\mathbf{B S})^{-1} \mathbf{T}_{1}$ is a diagonal matrix resulting in the control decoupling of pitch, roll and yaw axes. Application of this to our aircraft model with $K_{a r i}=1.66 \mathrm{deg} / \mathrm{deg}$, $K_{a e i}=0.75 \mathrm{deg} / \mathrm{deg}$, and $K_{r e i}=0.27 \mathrm{deg} / \mathrm{deg}$ results in the following decoupled equations:

$$
\begin{gathered}
-16.7 \dot{q}=-13.6 \alpha+10.9 q+\delta_{\text {pitch }} \\
-5 \dot{p}_{s}=67.9 \beta+7.7 p_{s}-5.8 r_{s}+\delta_{\text {roll }} \\
-46.8 \dot{r}_{s}=-222 \beta-10.3 p_{s}+22.1 r_{s}+\delta_{\text {yaw }}
\end{gathered}
$$

The above equations show the dependence of the rotational rates on the relevant aircraft states and the control inputs. The states $\alpha$ and $\beta$ are in radians, and the rates are in $\mathrm{rad} / \mathrm{s}$. It is noted that the state dependent terms appearing on the right hand sides are already stabilizing and therefore, we do not propose to cancel them.

In keeping with the main result in the previous section we propose the pitch control law as:

$$
\delta_{\text {pitch }} \cong-16.7 \dot{q}_{c m d}+K_{q}\left(q_{c m d}-q\right)
$$

Where, $q_{c m d}$ is the commanded pitch rate input. We substitute the pitch control law given by (35) into (32) and note that the gain $K_{q}=-105 \mathrm{deg} / \mathrm{rad} / \mathrm{s}$ will dominate the other terms in the right hand side. This results in the desired first order response of the aircraft pitch rate to a step input pitch rate command:

$$
\left(\dot{q}_{c m d}-\dot{q}\right) \cong \frac{K_{q}}{16.7}\left(q_{c m d}-q\right)
$$

It is to be noted that this value of $K_{q}$ was chosen to ensure that the actuators do not rate limit during a high gain maneuver like the auto landing. The effective time constant of the first order response works out to be: 


$$
\tau=\frac{-16.7}{-105}=0.16 s
$$

The actuators have a time constant of 0.05 s (i.e., 20rad/sec). Thus, the time scale separation between the actuator bandwidth and the inner loop bandwidth is about three times. Similarly, the roll and yaw axis inner loop control laws can be simplified as:

$$
\begin{aligned}
& \delta_{\text {roll }} \cong-5 \dot{p}_{\text {scmd }}+K_{p_{s}}\left(p_{\text {scmd }}-p_{s}\right) \\
& \delta_{\text {yaw }} \cong-46.8 \dot{r}_{\text {scmd }}+K_{r_{s}}\left(r_{\text {scmd }}-r_{s}\right)
\end{aligned}
$$

The roll gain is chosen to be $K_{p s}=-25 \mathrm{deg} / \mathrm{rad} / \mathrm{s}$ resulting in the first order response time constant of $0.2 \mathrm{~s}$, and the yaw gain is chosen to be $K_{r s}=-180 \mathrm{deg} / \mathrm{rad} / \mathrm{s}$ resulting in the first order response time constant of 0.26 s respectively.

The sampling rate of the feedback signals has been set at $50 \mathrm{~Hz}$ which gives us a Nyquist frequency of $157 \mathrm{rad} / \mathrm{sec}$. This is more than seven times the actuator bandwidth and is therefore acceptable from a time scale separation perspective. The sampling rate of the control computer is modeled by appropriate Pade approximations when we compute the stability margins.

Finally, for the inner most loops, we need to compute the angular accelerations $\dot{q}_{c m d}, \dot{p}_{\text {scmd }}, \dot{r}_{\text {scmd }}$. We choose to use washout filters set to a corner frequency 10 times the individual loop bandwidth. The phase lead at the loop bandwidth is then about 5degrees below the ideal value of 90degrees.

\section{Wind Axis Equations:}

The wind-axis system is used to define dynamics of the slow states. We follow [25] to recast the right hand sides of (18) in a simple form:

$$
\begin{gathered}
\dot{\mu}=p_{s} \operatorname{Cos} \beta+(q-\dot{\alpha}) \operatorname{Sin} \beta+\dot{\chi} \operatorname{Sin} \gamma \\
\dot{\alpha}=q-p_{s} \operatorname{Tan} \beta-\frac{(L-M g \operatorname{Cos} \mu \operatorname{Cos} \gamma)}{M V \operatorname{Cos} \beta} \\
\dot{\beta}=-r_{s}+\frac{g}{V} \operatorname{Sin} \mu \operatorname{Cos} \gamma-\frac{Y}{M V}
\end{gathered}
$$

In flight control, the intent is to achieve decoupling between angle-of-attack and sideslip by rolling about the velocity vector. The variable $\mu$ correctly captures the angle of roll about the velocity vector. The novelty in our formulation in (40-42) lies in using the stability axis angular rates in the right hand side. For the outer loop control design of the autopilot, we assume that the flight path angle $(\gamma)$, velocity roll angle $(\mu \leq 45 \mathrm{deg})$, and sideslip angle $(\beta)$ are small and slow compared to the states $\left(p_{s}, q, r_{s}\right)$. Further, we assume that lift balances weight, and the side force $Y$ is negligible. These assumptions lead to the following approximate dynamics:

$$
\begin{gathered}
\dot{\alpha} \cong q \\
\dot{\mu} \cong p_{s} \\
\dot{\beta} \cong-r_{s}+\frac{g}{V} \cdot \mu
\end{gathered}
$$


It is seen that in the above three equations, the derivatives of the state variables with respect to the pseudo control variables is 1,1 and - 1 respectively, making these derivatives independent of any specific aircraft. The outer loop control law based on the above approximations is given by:

$$
\begin{gathered}
q_{c m d}=\dot{\alpha}_{c m d}+K_{a l p h a}\left(\alpha_{c m d}-\alpha\right) \\
p_{s c m d}=\dot{\mu}_{c m d}+K_{m u}\left(\mu_{c m d}-\mu\right) \\
r_{\text {scmd }}=-\left[\dot{\beta}_{r e f}+K_{\text {beta }}\left(\beta_{r e f}-\beta\right)-\frac{g}{V} \cdot \mu\right]
\end{gathered}
$$

The gains are chosen to be: $K_{\text {alpha }}=2.0 \mathrm{rad} / \mathrm{s} / \mathrm{rad}, K_{m u}=3.0 \mathrm{rad} / \mathrm{s} / \mathrm{rad}$ and $K_{\text {beta }}=1.0 \mathrm{rad} / \mathrm{s} / \mathrm{rad}$, resulting in the time constants of $0.5 \mathrm{~s}, 0.33 \mathrm{~s}$, and $1.0 \mathrm{~s}$. It is to be noted that the outer loop time constants are at least 2.5 times of the inner loop time constants ensuring a significant dynamic separation between the cascaded loops. Again $\dot{\alpha}_{c m d}, \dot{\mu}_{c m d}$ are computed by using wash out filters with their respective corner frequencies set to 10 times the design loop bandwidth. The derivative of sideslip angle is zero.

\section{Velocity Vector Equations:}

The magnitude and direction of the velocity vector in are defined by (19). The states $(V, \gamma, \chi)$ are the very slow states. In the classical control design, the angles $(\gamma, \chi)$ are frequently replaced by the angles $(\theta, \psi)$ which together with the bank angle $\phi$ form the Euler angle triad. For the trajectory tracking autopilot, we make the reasonable assumption that the angles $\alpha, \beta, \gamma, \mu$ are small and the side force $Y$ is negligible (i.e., turns are coordinated), resulting in the first order approximations for (19) shown below:

$$
\begin{gathered}
\dot{V} \cong \frac{(T-D)}{M}-g \cdot \gamma \approx b_{v t h r} \cdot \delta_{t h r}-g \cdot \gamma \\
\dot{\gamma} \cong \frac{(L-M g)}{M V} \approx \frac{q b a r \cdot S C_{L \alpha}}{M V}\left(\alpha-\alpha_{t r i m}\right) \\
\dot{\chi} \cong \frac{L \cdot \mu}{M V} \approx \frac{g}{V} \mu
\end{gathered}
$$

In the above equations, $b_{v t h r}$ is the gradient of aircraft acceleration equation with respect to throttle deflection, and appears in the B-matrix as:

$$
b_{v t h r}=\frac{\partial \dot{V}}{\partial \delta_{t h r}}
$$

Further, $C_{L \alpha}$ is the gradient of the lift curve slope, $\alpha_{t r i m}$ is the trim angle of attack, and qbar is the dynamic pressure. It is seen that (49) and (50) have parameters which are aircraft specific, namely derivative of velocity rate with respect to throttle and the derivative of lift force with respect to angle of attack respectively. Equation (50) conveys that for a fixed wing aircraft the curvature of the flight path in the vertical plane is achieved by increasing the lift force through changing the angle of attack. Similarly, (51) indicates that the flight path curvature in the horizontal plane is achieved by tilting the lift vector in the direction of the turn. 
The control law for the tracking loop, based on the above approximations, is given by:

$$
\begin{gathered}
\delta_{t h r}=\frac{1}{b_{v t h r}}\left[\dot{V}_{r e f}+K_{v e l}\left(V_{r e f}-V\right)+g \cdot \gamma\right] \\
\alpha_{c m d}=\frac{M V}{q b a r \cdot S C_{L \alpha}}\left[\dot{\gamma}_{c m d}+K_{g a m}\left(\gamma_{c m d}-\gamma\right)\right]+\alpha_{t r i m} \\
\mu_{c m d}=\frac{V}{g}\left[\dot{\chi}_{c m d}+K_{c h i}\left(\chi_{c m d}-\chi\right)\right]
\end{gathered}
$$

The gains are chosen to be: $K_{v e l}=0.5 \mathrm{~s}^{-1}, K_{\text {gam }}=1.2 \mathrm{rad} / \mathrm{s} / \mathrm{rad}$ and $K_{c h i}=0.5 \mathrm{~s}^{-1}$. These values result in first order time constants of $2 \mathrm{~s}, 0.8 \mathrm{~s}$, and $2 \mathrm{~s}$ respectively for these loops. The time constants of the flight path and heading angle loops are at least 2.5 times those of the angle of attack and bank angle loops thereby ensuring adequate dynamic separation. The derivatives of the pseudo control signals $\dot{V}_{r e f}, \dot{\gamma}_{c m d}, \dot{\chi}_{c m d}$ are constructed by passing them through washout filters with corner frequency 10 times the respective loop bandwidths.

\section{Navigational Equations:}

Finally, the navigational equations are given in the usual format in (20). The first order approximations for the equations for lateral and vertical deviations are given by:

$$
\begin{gathered}
\dot{y} \cong V \cdot\left(\chi_{\text {cmd }}-\chi_{\text {ref }}\right) \\
\dot{h} \cong V \cdot \gamma
\end{gathered}
$$

It is noted that there are no aircraft specific parameters in these equations. The control law for the position loop, based on the above approximations, is given by:

$$
\begin{gathered}
\left(\chi_{c m d}-\chi_{r e f}\right)=\frac{1}{V}\left[\dot{y}_{r e f}+K_{y}\left(y_{r e f}-y\right)\right] \\
\gamma_{c m d}=\frac{1}{V}\left[\dot{h}_{r e f}+K_{h}\left(h_{r e f}-h\right)\right]
\end{gathered}
$$

The gains are chosen to be: $K_{y}=0.1 \mathrm{~s}^{-1}$ and $K_{h}=0.65 \mathrm{~s}^{-1}$. These values result in first order time constants of $10 \mathrm{~s}$ and $1.5 \mathrm{~s}$ respectively for these loops. The time constants of the cross track and the altitude loops is at least 1.9 times those of the bank angle and flight path angle loops thereby ensuring adequate dynamic separation between the cascaded loops. The derivative of the $y_{\text {ref }}$ signal is zero. The derivative of the altitude signal is computed using the washout filter with time constant 10 times the loop bandwidth.

The angle of attack and sideslip angle require a calibrated and fail safe air data system to measure them accurately. Therefore, if such signals are not available, a possible alternative to these signals are the nearly equivalent signals of normal acceleration and lateral acceleration respectively:

$$
\alpha=\left(\frac{1}{n_{\alpha}}\right) n_{z}
$$




$$
\beta=\left(\frac{1}{n_{\beta}}\right) n_{y}
$$

The angle of attack and sideslip feedback loops can be implemented computing their values from normal acceleration and lateral acceleration feedback signals as per (60) and (61) respectively. The corresponding gains for the angle of attack and sideslip computed by the procedure above may have to be reduced marginally to achieve acceptable closed loop response.

In a similar manner we note that the rate of change of Euler angles $(\phi, \theta, \psi)$ is defined as:

$$
\begin{aligned}
& \dot{\phi}=p+(q \operatorname{Sin} \phi+r \operatorname{Cos} \phi) \operatorname{Tan} \theta \\
& \dot{\theta}=q \operatorname{Cos} \phi-r \operatorname{Sin} \phi \\
& \dot{\psi}=(q \operatorname{Sin} \phi+r \operatorname{Cos} \phi) \operatorname{Sec} \theta
\end{aligned}
$$

In the first approximation assuming small pitch and bank angles $(\phi, \theta)$, one can write these relations as:

$$
\begin{aligned}
& \dot{\phi} \cong p \\
& \dot{\theta} \cong q \\
& \dot{\psi} \cong r
\end{aligned}
$$

The above equations suggest that the variables $(\mu, \alpha, \chi)$ used in state feedback can be replaced by the set of variables $(\phi, \theta, \psi)$ in that order to obtain the typical classical feedback structure. The gains computed for the former set can be retained for the latter controller as a first guess.

\section{Comparison Between Classical and DDBS Autopilots}

\section{A. Classical Controller Design Method}

The classical design is undertaken in two phases separately; one for longitudinal axis and another for lateral-directional axis. All the loops have been designed with a minimum gain margin of $6 \mathrm{~dB}$ and a phase margin of at least $45 \mathrm{deg}$.

\section{Longitudinal Axis}

The innermost pitch rate loop is designed first followed by the pitch attitude loop. A washout filter in the pitch attitude feedback signal path is designed to remove the steady-state component of signal. The velocity to throttle feedback loop is designed with a lead-lag compensator in the forward path of this loop. The flight path angle feedback with lag-lead filter brings in all the RHP poles into the LHP. The altitude loop is closed with a proportional-integral gain and an altitude rate feed forward term as shown in Fig. 2.

\section{Lateral-directional Axis}

The innermost loop in the lateral axis is the roll rate loop designed for achieving crisp roll rate response. The yaw rate feedback in the innermost loop in the directional axis is designed to improve the damping of the Dutch Roll mode and suppress sideslip development. Examination 
of the equations of motion, indicate that the sideslip rate can be approximated by two components. The first is the term $(r-p \operatorname{Tan} \alpha)$ and the other is the lateral acceleration. Since, it is our intention to minimize the sideslip, a combination of these two quantities is used. The ( $\operatorname{Tan} \alpha$ ) is approximated as 0.2 , corresponding to the 1 -g value of the trim angle of attack. This feedback loop increases the damping while having negligible effect on the roll mode. The bank angle feedback moves the spiral mode poles to the LHP. Finally, a PID design is attempted on the track angle deviation. The integral of track angle deviation is proportional to the track deviation in meters. The derivative part is replaced with a feed forward correction using the reference track angle deviation. Aileron to rudder interconnect gain of $1.2 \mathrm{deg} / \mathrm{deg}$ is introduced to provide an open loop sideslip reduction. The lateral-directional scheme is as shown in Fig. 3. In this figure, we have also introduced a PID which is switched into the rudder channel when the altitude drops below 2meters, while the feedback signal to the outermost loop switches from being the track angle to the heading angle simultaneously. This is to reduce the relative heading angle between the runway and the aircraft before touchdown.

In Figs. 2 and 3, we have included a control allocation block. This block is designed subsequent to the longitudinal and lateral-directional control design to exploit as much as possible the redundancy in the fight controls. Since, the aileron to rudder interconnect is already accounted for the ganging matrix is as follows:

$$
\mathbf{u}=\left[\begin{array}{ccc}
1 & -0.75 & -0.1 \\
1 & 0.75 & 0.1 \\
0 & -1 & 0 \\
0 & 1 & 0 \\
0 & 0 & 1
\end{array}\right]\left[\begin{array}{c}
\delta_{\text {pitch }} \\
\delta_{\text {roll }} \\
\delta_{\text {yaw }}
\end{array}\right]
$$

The values of 0.75 and 0.1 were arrived at by a process of trial and error in this case to give best results for the failure tolerance envelope in connection with single or two surface failure events [26].

The trial and error involved in arriving at the gains as well as the ganging matrix for the classical control design is significantly high compared to the systematic procedure outlined for the DDBS even though both the controllers are arrived at by loop by loop design process. Furthermore, the time scale separation allows us to arrive at a closed form solution for the controller gains in case of the latter. This advantage becomes more pronounced for aircraft where the autopilot has to be designed throughout the flight envelope and a gain schedule is required for this purpose.

\section{B. Diagonally Dominant Backstepping Controller}

The schematic of the DDBS control law, developed in the present work, is shown in Fig. 1. The cascaded structure of the control law is chosen such that the dynamic inversion parts of the control law fit into a classical inner loop - outer loop structure. The margins for each loop are a minimum gain margin of $6 \mathrm{~dB}$ and a phase margin of at least $45 \mathrm{deg}$.

Apart from the DDBS control concepts discussed above, a few additional control features are implemented by us in the control law. All the pseudo control signals are constructed using washout filters with corner frequency 10 times the respective loop bandwidth. The air speed loop also has an additional lead-lag compensator to improve the speed of response without compromising the overshoot. The longitudinal and lateral-directional closed loop control 
schemes are shown in Figs. 4 and 5 respectively. These figures also have standard PID loops in some of the outer loops. The proportional gain chosen for these loops is same as that computed above. The integral gain is about $5 \%$ of the proportional gain in both axes. No derivative gains were required for this aircraft.

\section{Anti-windup and State Limiting}

High gains can result in saturations due to control surface limits. Antiwindup and adaptive schemes are available in literature for nonlinear dynamic inversion controllers ([27], [28]). The anti-windup protection in our controller is developed as follows. To protect against integrator windup, control surface saturation is detected. The control allocation matrix in (23) means that for example if the left elevator is saturated, it could be due to either the pitch, roll or yaw axis control signals. Therefore, in this case we hold the integrators in all the three axes for the duration for which the left elevator is saturated. This way we prevent the control system from overdriving the actuators in Figs. 4 and 5.

It is also seen that the limits on the state variables like pitch attitude is also incorporated in the pitch axis integrator. The general principle for the anti-windup design is that any state or control surface saturation in the inner loops of the cascaded controller should result in the integrators in the loops outer to be held for the duration of the time the variable is in saturation. Thus, in Fig. 4 we see that if the angle of attack command is saturated, this results in the integrator in the altitude PID being held for that duration. This general anti-windup scheme addresses both state and control surface saturation for a cascaded controller structure.

\section{Comparison}

It is instructive to compare the longitudinal design using classical loop shaping presented in Fig. 2 with the DDBS design derived in Fig. 4. Firstly, both the designs have a cascade structure. The classical design structure is the result of loop by loop single-input single-output design approach typically adopted for autopilots. On the other hand, the DDBS controller has a cascade structure due to the block triangular form of the equations of motion. Similar conclusions are valid for the lateral-directional controllers designed by the classical approach (Fig. 3) and DDBS approach (Fig. 5).

The other difference relates to the feedback variables used in the two designs. The classical design assumes that air data sensors like angle of attack and sideslip are not available. Therefore, in Fig. 2 pitch attitude is used in place of the angle of attack in the longitudinal axis. Similarly, the lateral-directional design in Fig. 3 uses bank angle for feedback as opposed to velocity vector roll angle in Fig. 5. The gravity related compensation terms seen in Figs. 4-5 is a natural outcome of the DDBS control design. These terms are absent from the classical controller in Figs. 2-3.

We find that between the classical design and the DDBS design, there are loops which are common. The steady-state gains for the common loops obtained by these two designs methods are identical in some cases (e.g., tracking loop gain) and comparable in other cases (e.g., flight path angle gain). In fact, the authors have verified that one can take the DDBS design as the baseline structure and systematically replace individual feedback signals by their equivalent signals within a scale factor (e.g., angle of attack by pitch attitude, angle of attack by normal acceleration, sideslip by lateral acceleration, velocity vector angle by bank angle etc.) and still obtain good closed loop performance without changing the DDBS gains. The baseline DDBS controller gains can also be supplemented by series compensators like the lead-lag present in the 
velocity loop to obtain further improvement (Fig. 4). Therefore, in conclusion, a first estimate of the gains for the cascaded controller can be obtained by following DDBS design procedure outlined in the previous section where either state feedback or output feedback controller is to be designed.

The classical and DDBS controllers were evaluated for parametric uncertainties by varying the mass by $20 \%$ of nominal value, inertia by $10 \%$ of nominal value and center of gravity variation from $30 \%$ to $40 \%$ of mean aerodynamic chord. Both the controllers demonstrated adequate performance for these variations. The results are not presented here due to lack of space.

Performance guarantees are not included in the design process for either classical approach or the DDBS controller design presented here. The gains in these designs are selected to be as high as permissible at each stage keeping in mind the actuator constraints and the time scale separation principle. Therefore, the stability and performance robustness is an outcome of the design process. In practice, if the uncertainties are larger it is possible that even stable systems may suffer from significant performance degradation.

The DDBS design approach described in this paper has the following benefits:

1. Classical gain and phase margins required for certification can be provided for any fixedwing air vehicle including unmanned aerial vehicles.

2. A method of handling control surface saturation and state vector limiting for the DDBS controller is developed.

3. A novel control decoupling scheme for the innermost loops is developed which simultaneously achieves fault tolerance for two aileron failure. This scheme requires that the left and right elevator can be deflected separately which is unlike that used in a conventional flight control system.

4. In case all the signals required for full state feedback are not available, alternate signals normally used for flight control design can also be incorporated into the design.

5. The DDBS controller can be improved further by using classical loop shaping techniques to target performance metrics like speed of response.

6. The loop gains are obtained in a natural manner based on the separation of time scales principles and literal expressions derived from the model formulation. This is a direct benefit of the dynamic inversion and is in contrast to the classical loop shaping approach where the designer typically discovers the gain value by trial and error.

7. Time scale separation ensures that outer loop bandwidth is low to prevent severe atmospheric disturbances causing control surface rate or position saturation in the inner loops. At the same time the inner loop gains are high so as to overcome a large class of unknown control surface failures.

\section{Simulation Results}

The performance and robustness characteristics of the DDBS controller are illustrated using a six-degree-of-freedom simulation of the autolanding task shown in Fig. 6 [26]. The autolanding scenario consists of level flight segments, level turns, descent profiles and the flare and touchdown maneuvers. A tracking command generator provides reference commands to the controller based on trajectory deviation of the aircraft. The reference commands consist of altitude reference $\left(h_{r e f}\right)$, velocity reference $\left(V_{\text {ref }}\right)$, cross distance from the desired track $\left(y_{\text {ref }}-y\right)$, and the angular error of the aircraft velocity vector from the desired track vector $\left(\chi_{\text {ref }}\right)$. The 
autolanding control system is designed to land the aircraft on the runway within a rectangular area or 'pillbox' measuring $10 \mathrm{~m}$ in width, and $400 \mathrm{~m}$ in length, with $V \geq 60 \mathrm{~m} / \mathrm{s}, \dot{h} \leq-1 \mathrm{~m} / \mathrm{s}$, $|\psi| \leq 15 \mathrm{deg}$, and $|\phi| \leq 10 \mathrm{deg}$ to avoid stall, damages to the landing gear, and wing tips respectively.

The wind profiles used during autolanding are shown in Fig. 7. The wind disturbances are based on the Dryden model along the North and severe wind shear and micro burst along the East and Down axes respectively. The autolanding simulations were carried out under normal operating conditions without wind disturbances, and under external wind disturbances and unknown actuator failures.

The simulation results for the linear longitudinal and lateral-directional model to a step and ramp inputs for the classical controller design are shown in Figs. 8-9 respectively. Similarly, the results for the longitudinal and lateral-directional DDBS controller are shown in Figs. 10-11 respectively. Comparing Figs. 8 and 10, we find that the DDBS controller has a faster response to step input to a speed command compared to the classical controller. The linear lateraldirectional responses to a ramp have been plotted in Figs. 9 and 11 respectively for the classical and DDBS controller. The sideslip response for the DDBS controller is particularly small during the ramp following due to the full control decoupling as well as explicit beta feedback. The DDBS controller also demonstrates more undershoot in the cross track deviation. This is expected based on the control structure used in the two designs. In case of the classical design, we have added the cross track error path directly to the bank angle, while in case of the DDBS controller it is added to the reference heading command.

Figs. 12-13 show the simulation results for autolanding under severe wind disturbances and with left-elevator stuck at $14 \mathrm{deg}$ at 35seconds. We immediately note in Fig. 12 that the deflection limits of the healthy control surfaces (elevator $\pm 25 \mathrm{deg}$, aileron $\pm 20 \mathrm{deg}$ and rudder $\pm 30 \mathrm{deg})$ are being hit repeatedly due to winds in segments 6 and 7. Due to the left elevator failure at about 35 seconds, the mean value of the right elevator has moved in the opposite direction to compensate the unbalanced pitching moments. The ailerons are deflected to compensate the resulting roll moment due to differential elevators. The controller is able to restrict the angle of attack within limits in segments 2 and 7. We also note that the touchdown point is closer to the $5 \mathrm{~m}$ lateral boundary. In Fig. 13 we find that momentary rate limiting ( $\pm 60 \mathrm{deg} / \mathrm{sec}$ ) of the healthy actuators is seen. However, the terminal values of the sink rate, bank angle and heading angle at touchdown are within acceptable limits.

The simulation results for simultaneous failure of both the ailerons at 33seconds are shown in Figs. 14-15. We see from Fig. 14 that there is a significant amount of mean sideslip (about 6degrees) and corresponding rudder deflection due to loss of both ailerons. The healthy elevators are taking up the unbalanced rolling moment through differential deflection. The angle of attack is successfully limited to within the limits. Actuator rate limiting is more prominent in Fig. 15 as compared to Fig. 13. Finally, it can be seen from these figures that the controller meets all the performance and safety requirements specified.

Another controller was designed by replacing the angle of attack and sideslip signals in the DDBS controller (Figs. 4-5) using normal and lateral acceleration feedback as per (60) and (61) respectively. Figures 16-17 show the results of simulating the 14deg elevator stuck failure case with this controller. It is seen in Fig. 16 that although this controller is able to successfully complete the autolanding task within the pillbox meeting the safety requirements, both the angle of attack and the sideslip show large deviations during the first turn (segment 2). The angle of 
attack which is not an active feedback variable does in fact exceed the maximum limit of 25deg for a while. In later segments however, the angle of attack does not show such large excursions. Therefore, performance of the angle of attack limiter using the antiwindup concept proposed above while using the normal acceleration for feedback is not consistent. In Fig. 17 we note that the healthy elevator and the rudder actuators show increased rates during the flight for this controller. In addition, significant overshoots are seen in the bank angle during the turn in segments 2 and 4 particularly after the single elevator failure at $35 \mathrm{sec}$.

\section{Conclusions}

A new approach called Diagonally Dominant Backstepping has been developed in this paper for the design of trajectory following autopilots for fixed wing aircraft including unmanned air vehicles. This is a simplification of the well known block backstepping method of design for nonlinear aircraft control permitting systematic loop by loop design of autopilots for fixed wing aircraft.

Parametric studies in terms of variation of mass, inertia and center of gravity demonstrate that the design is robust to these uncertainties. Simulation results with elevator actuator stuck shows the robustness of the controller to the loss of elevator control power by $50 \%$. Similarly, the two aileron failure case presented in the results effectively implies $100 \%$ loss of aileron control power. In this case, it is use of elevators in a differential mode which provide adequate roll control. The design process also caters for requirement of output feedback as opposed to full state feedback by suggesting appropriate replacement of some of the state feedback signals with readily available equivalent output signals. A three control surface failure case (two ailerons and the rudder failed to a stuck position) has also been shown confirming the robustness of the design to multiple failures. The robustness against these parametric perturbations has been demonstrated for an auto-landing task when the aircraft is subject to severe winds.

\section{Acknowledgments}

The authors express their sincere thanks to the organizations NAL-CSIR Bangalore, and NIT Trichy for their encouragement throughout the course of this work. The authors also thank Dr. Srikanth Rao, Honeywell Technology Solutions Laboratories, Bangalore for his inputs during the course of preparation of this manuscript.

\section{References}

[1] Kato, O. and SugiURA, I. An interpretation of airplane general motion and control as inverse problem, J. of Guidance, March-April 1986, 9, (2), pp. 198-204.

[2] Krstic, M., KAnellakopoulos, I. and KoKotoviC, P.V. Nonlinear and Adaptive Control Design, New York, USA: John Wiley \& Sons, Inc., 1995.

[3] HARKEGARD, O. Backstepping and control allocation with applications to flight control, Ph.D. dissertation, Dept. Electrical Eng., Linkoping Univ., Linkoping, Sweden, 2003.

[4] OORT, van E.R. Adaptive backstepping control and safety analysis for modern fighter aircraft,” Ph.D. dissertation, Faculty of Aerospace Engineering, TU Delft, Netherlands, 2011. 
[5] ThunBerg, J. and RoBinson, J.W.C. Block backstepping, NDI and related cascade designs for efficient development of nonlinear flight control laws, AIAA-2008-6960, AIAA Guidance, Navigation and Control Conf. and Exhibit, 18-21 Aug. 2008, Honolulu, Hawai.

[6] KNOOS, J., RoBinson, J.W.C. and BerefELt, F. Nonlinear dynamic inversion and block backstepping: A comparison, AIAA-2012-4888, AIAA Guidance, Navigation, and Control Conf., 13-16 Aug. 2012, Minneapolis, Minnesota.

[7] SonneveldT, L., OORT, van E.R., ChU, Q.P., Mulder, J.A. Nonlinear adaptive trajectory control applied to an F-16 model, J. of Guidance, Control and Dynamics, January-February 2009, 32, (1), pp. 25-39.

[8] IsIDORI A. Nonlinear Control Systems, Springer-Verlag, Berlin, 1989.

[9] MeYer, G., Su, R. and HunT, L.R. Application of nonlinear transformations to automatic flight control, Automatica, 20, (1), January 1984, pp. 103-107.

[10] Menon, P.K.A., BAdgetT, M.E., Walker, R.A. and Duke, E.L. Nonlinear flight test trajectory controller for aircraft, J. of Guidance, Control, and Dynamics, Jan-Feb 1987, 10, (1), pp. 67-72.

[11] LANE, S.H. and STENGEL, R.F. Flight control design using nonlinear inverse dynamics, Automatica, July 1988, 24, (4), pp. 471-483.

[12] BugAJsKi, D.J. and EnNS, D.F. Nonlinear control law with application to high angle-ofattack, J. of Guidance ,Control, and Dynamics, May-June 1992, 15, (3), pp. 761-767.

[13] SNELL, S.A., ENNS, D.F. and GARRARD W.L. Jr. Nonlinear inversion control for a supermaneuverable aircraft, J. of Guidance, Control, and Dynamics, July-August 1992, 15, (4), pp. 976-984.

[14] MiLleR, C.J. Nonlinear dynamic inversion baseline control law: flight- test results for the full-scale advanced systems testbed F/A-18 airplane, AIAA-2011-6468, AIAA Guidance, Navigation, and Control Conference Portland Oregon USA, 2011.

[15] BRINKER, J.S. and WISE, K. Stability and flying qualities robustness of a dynamic inversion aircraft control law, J. of Guidance, Control, and Dynamics, November-December 1996, 19, (6), pp. 1270-1277.

[16] ADAMS, R.J. and BANDA, S.S. An integrated approach to flight control design using dynamic inversion and mu-synthesis, Proc. of the ACC, 1993, pp. 1385-1389.

[17] Buffington, J.M., AdAMS, R.J. and BANDA, S.S. Robust nonlinear high angle of attack control design for a supermaneuverable vehicle, Proc. of AIAA Guidance, Navigation, and Control Conf., 1993, pp. 690-700.

[18] GOMAN, M.G. and KOLESNIKOV, E.N. Robust nonlinear dynamic inversion method for an aircraft motion control, AIAA-98-4208, AIAA Guidance, Navigation and Control Conf. and Exhibit, 10-12 Aug. 1998, Boston M.A., USA.

[19] Reigelsperger, W.C., BANDA, S.S. and LEMAster, D.P. Application of multivariable control theory to aircraft control laws. Final Report - Multivariable control design guidelines, WL-TR-96-3099, Flight Dynamics Directorate, Wright Laboratory, Wright-Patterson AFB, OH, May 1996.

[20] Farrell, J., Sharma, M. and Polycarpou, M. Backstepping Based Flight Control with Adaptive Function Approximation, J. of Guidance, Control, and Dynamics, NovemberDecember 2005, 28, (6), pp. 1089-1102.

[21] Stein, G. Respect the unstable, IEEE Control Systems Magazine, 23, (4), August 2003, pp. $12-25$. 
[22] Nguyen, L.T., Ogburn, M.E., Gilbert, W.P., Kibler, K.S., Brown, P.W. and DeAl, P.L. Simulator study of stall/post-stall characteristics of a fighter airplane with relaxed longitudinal static stability, NASA TP-1538, 1979.

[23] TEO, S.G. Autolanding system study: Aerodynamic data of an aircraft with independent control surfaces by CFD, Technical Report of DSO National Lab., Singapore, 2003.

[24] PASHILKar, A.A., Sundararajan, N. and SARATCHANDRAn, P. Adaptive nonlinear neural controller for aircraft under actuator failures, J. of Guidance, Control, and Dynamics, May-June 2007, 30, (3), pp. 835-847.

[25] Miele, A. Flight Mechanics Volume I: Theory of Flight Paths. Addison-Wesley Pub. Co. Inc., 1962.

[26] Ismail, S., PAShilkar, A.A., Ayyagari, R. and Sundararajan, N. Improved autolanding controller for aircraft encountering unknown actuator failures, Proc. IEEE Symposium on Computational Intelligence for Security and Defense Applications (IEEE CISDA 2013), Singapore, 2013.

[27] ValaseK, J., AKella, M.R., Siddarth, A. and Rollins, E. Adaptive dynamic inversion control of linear plants with control position constraints, IEEE Transactions on Control Systems Technology, July 2012, 20, (4), pp.918-933.

[28] Menon, P. P., Lowenberg M., Herrmann, G., Turner, M. C. Bates, D. G., and PostlethWAite, I. Experimental implementation of a nonlinear dynamic inversion controller with antiwindup, J. of Guidance, Control, and Dynamics, July-August 2013, 36, (4), pp. 10351046. 


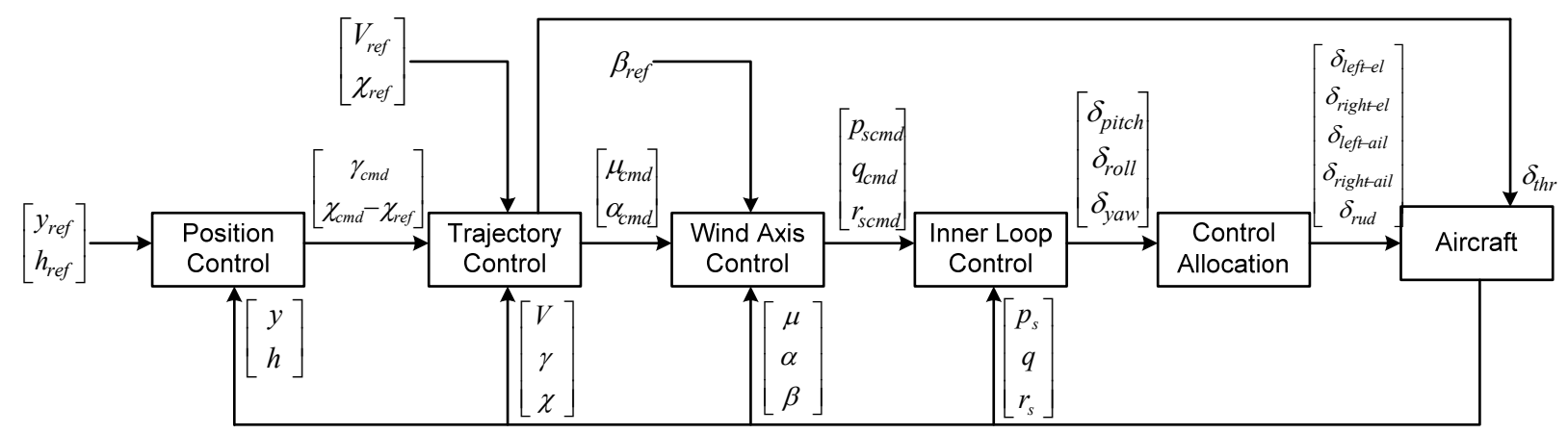

Fig. 1. Schematic of Controller based on the application of NDI principles. 


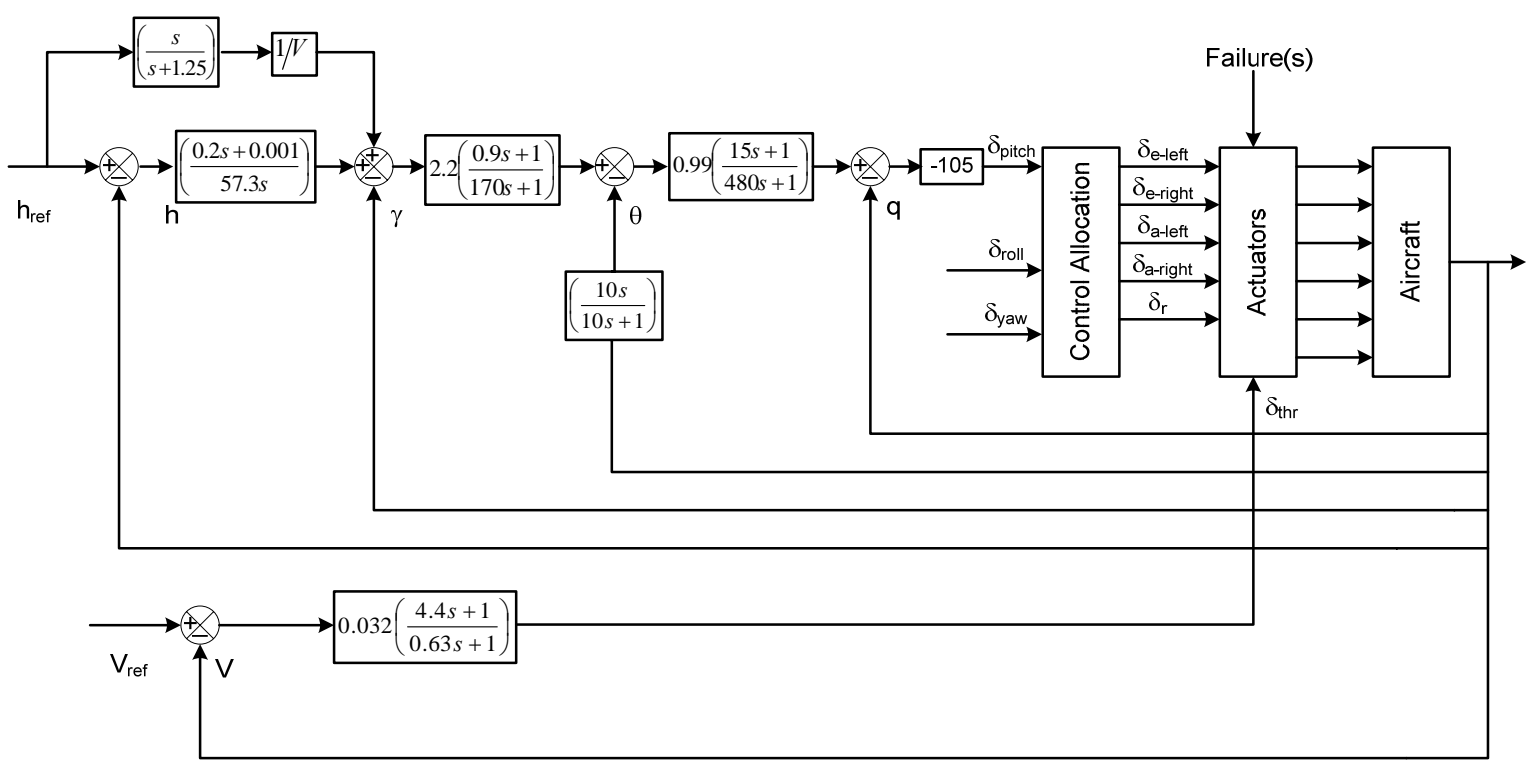

Fig. 2. Classical longitudinal axis controller. 


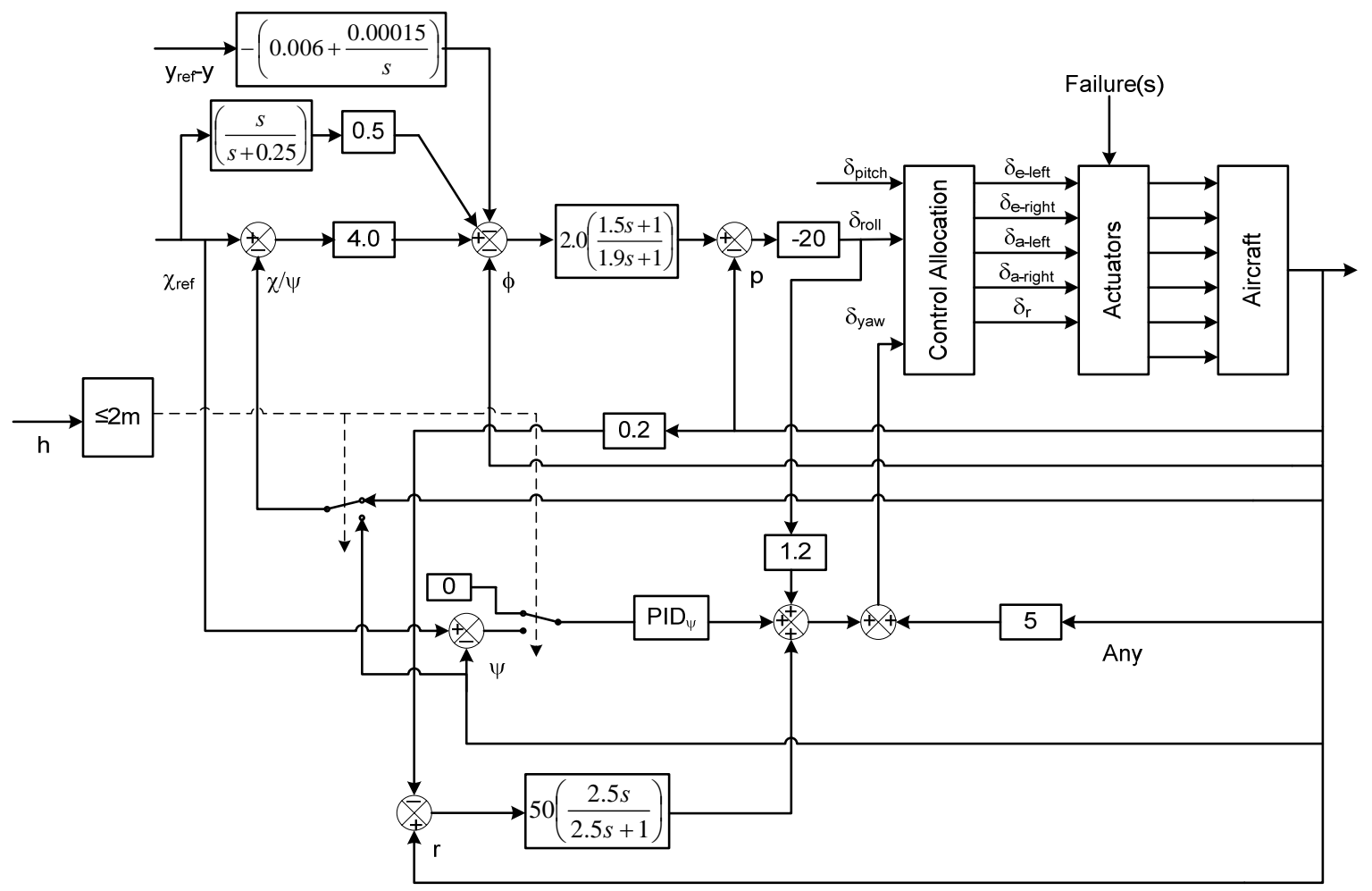

Fig. 3. Classical lateral-directional axis controller. 


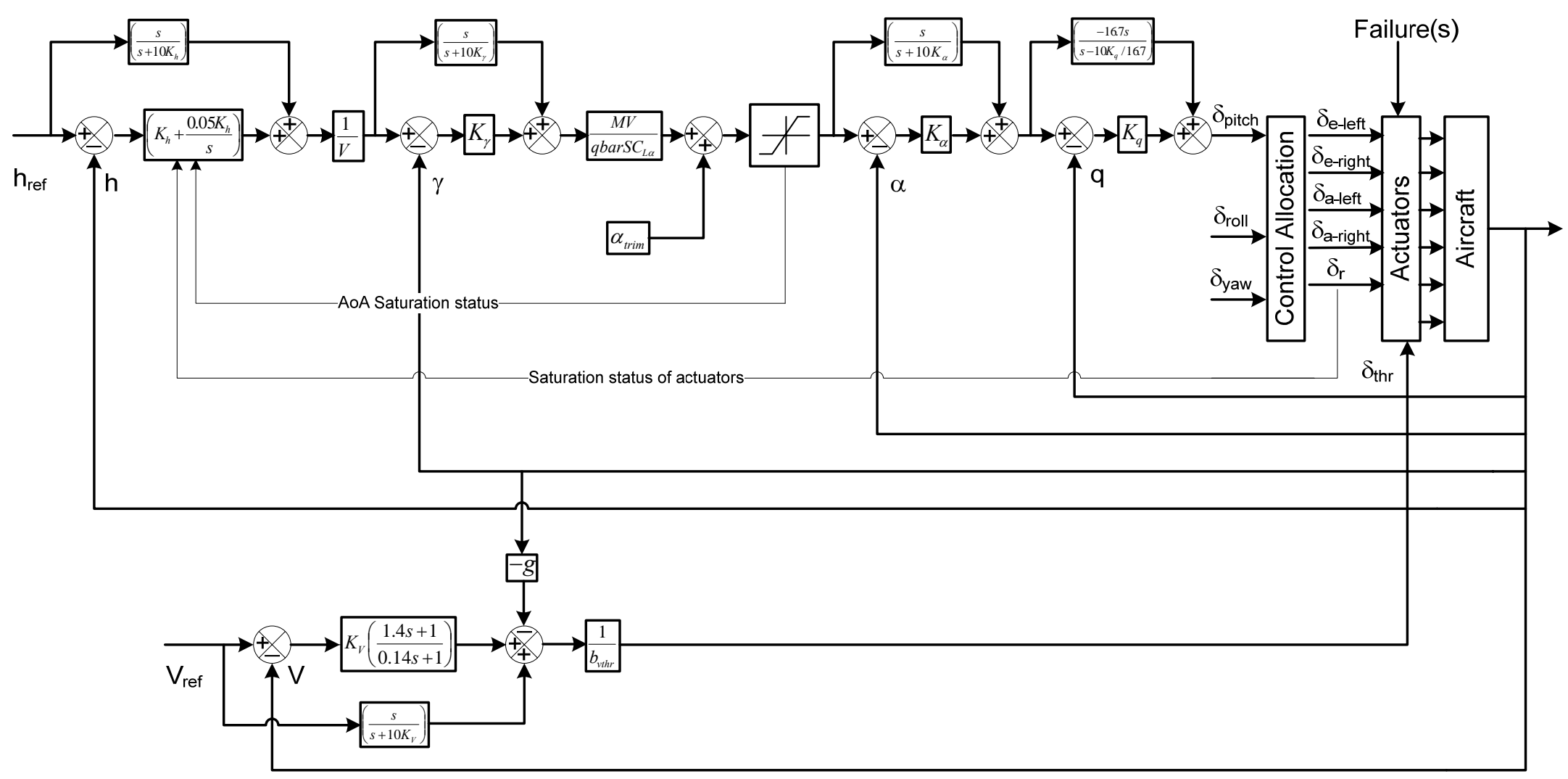

Fig. 4. Longitudinal axis DDBS Controller. 


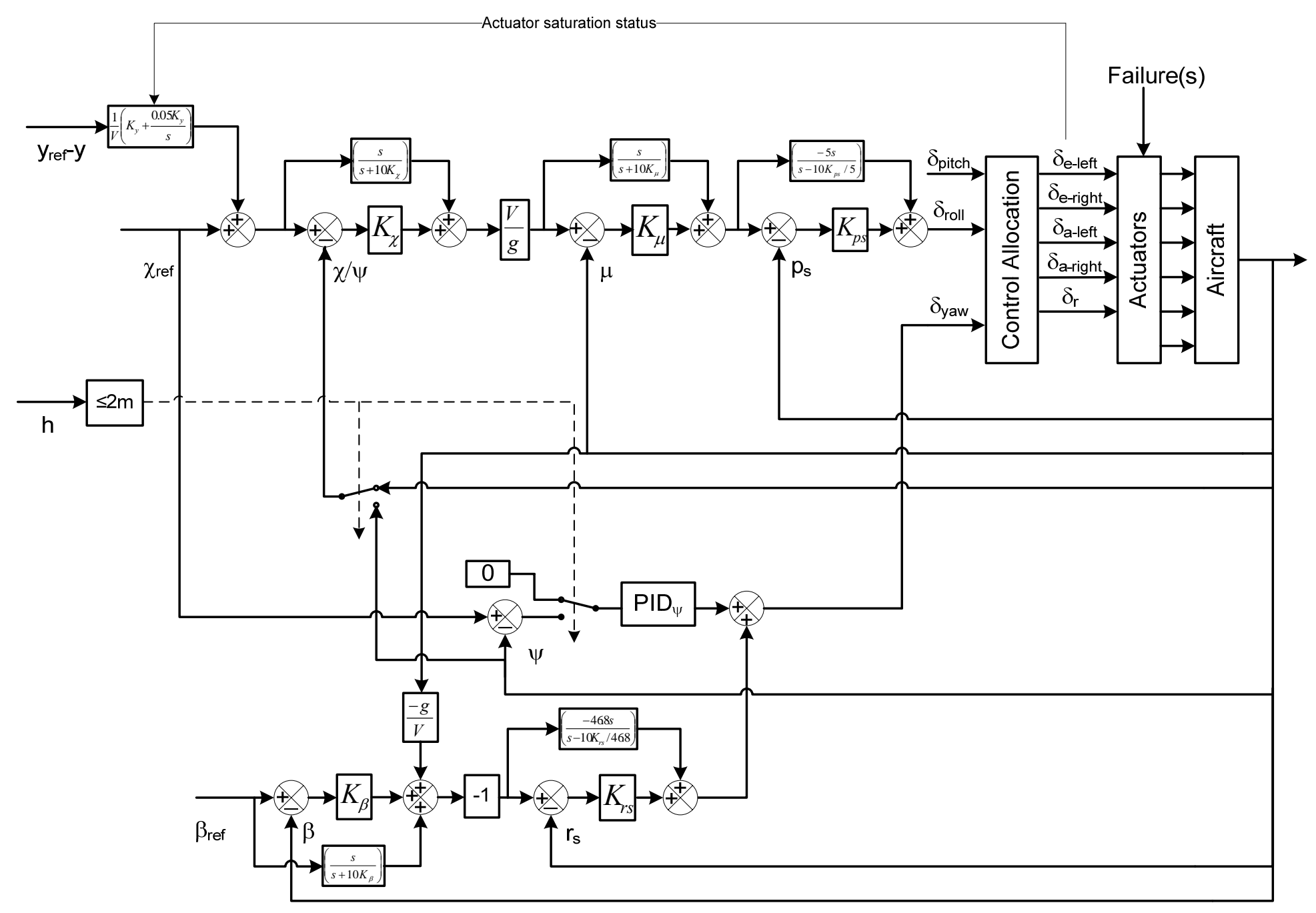

Fig. 5. Lateral-directional axis DDBS Controller. 


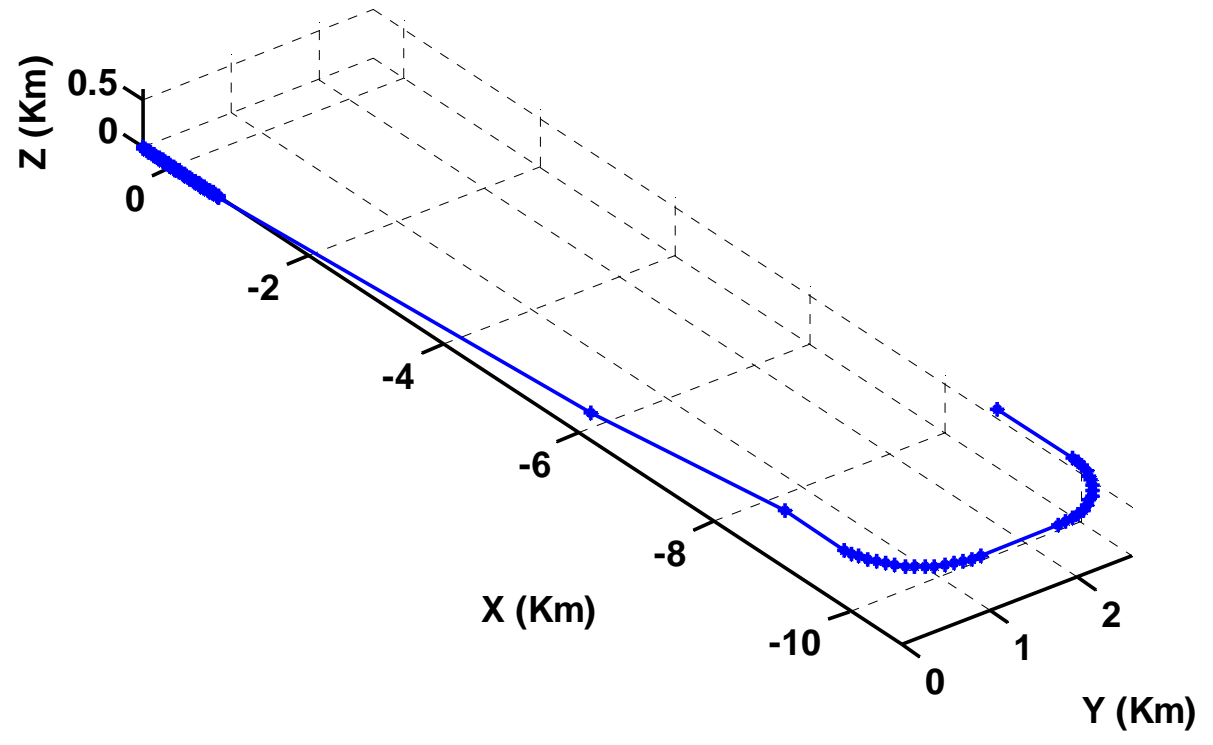

Fig. 6. Autolanding trajectory. 

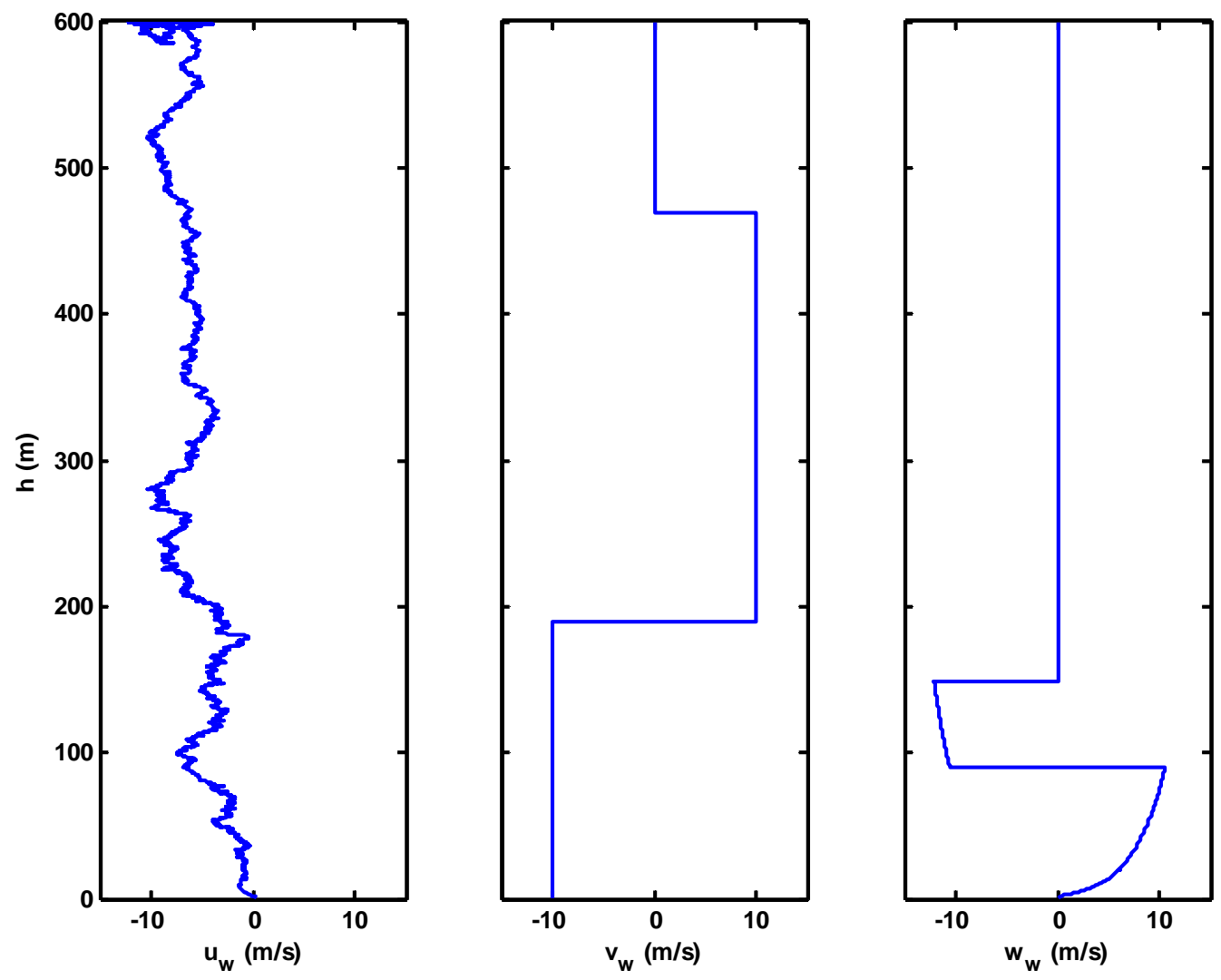

Fig. 7. Wind profiles during autolanding task. 

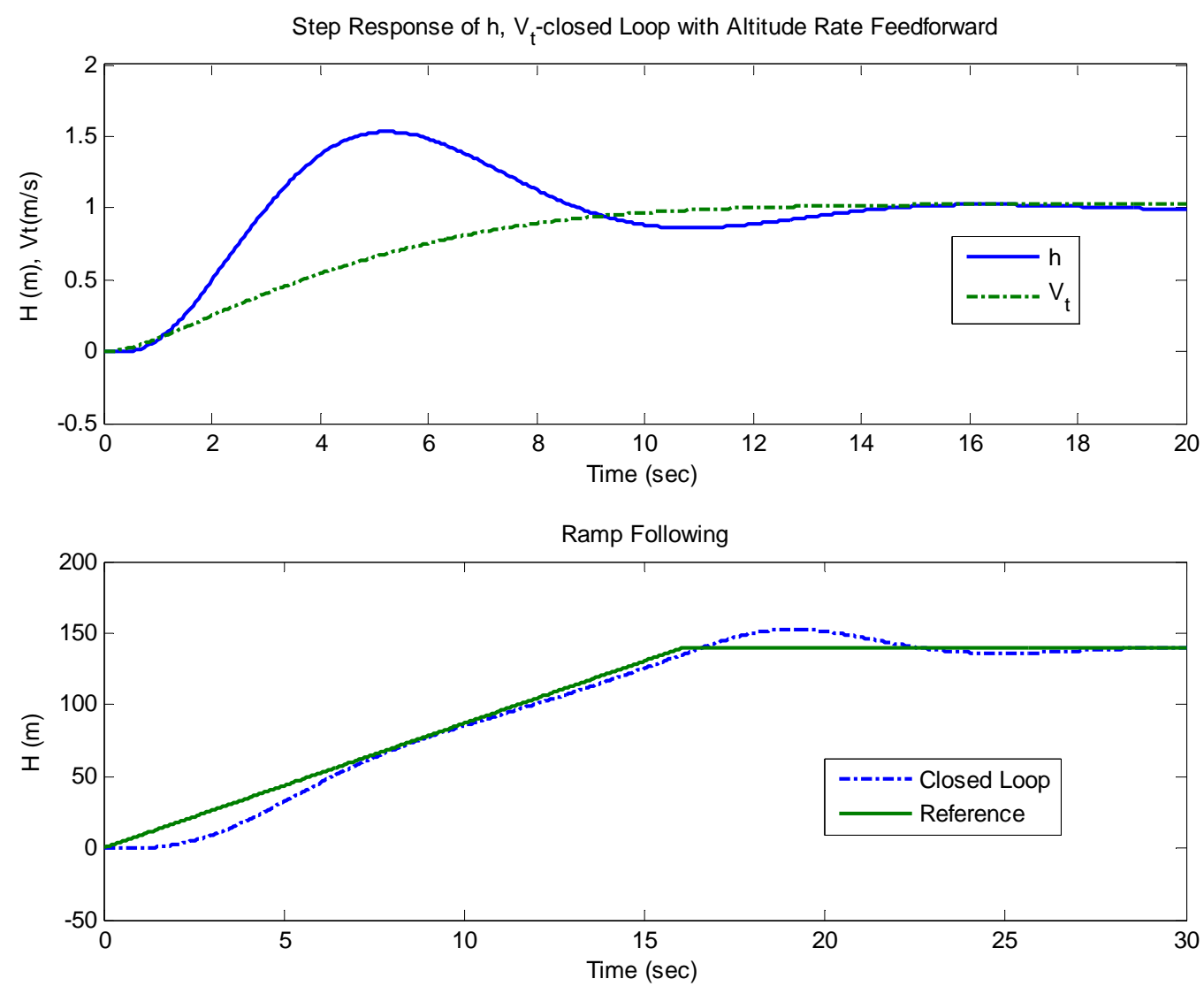

Fig. 8 Closed loop linear response of the classical longitudinal controller. 

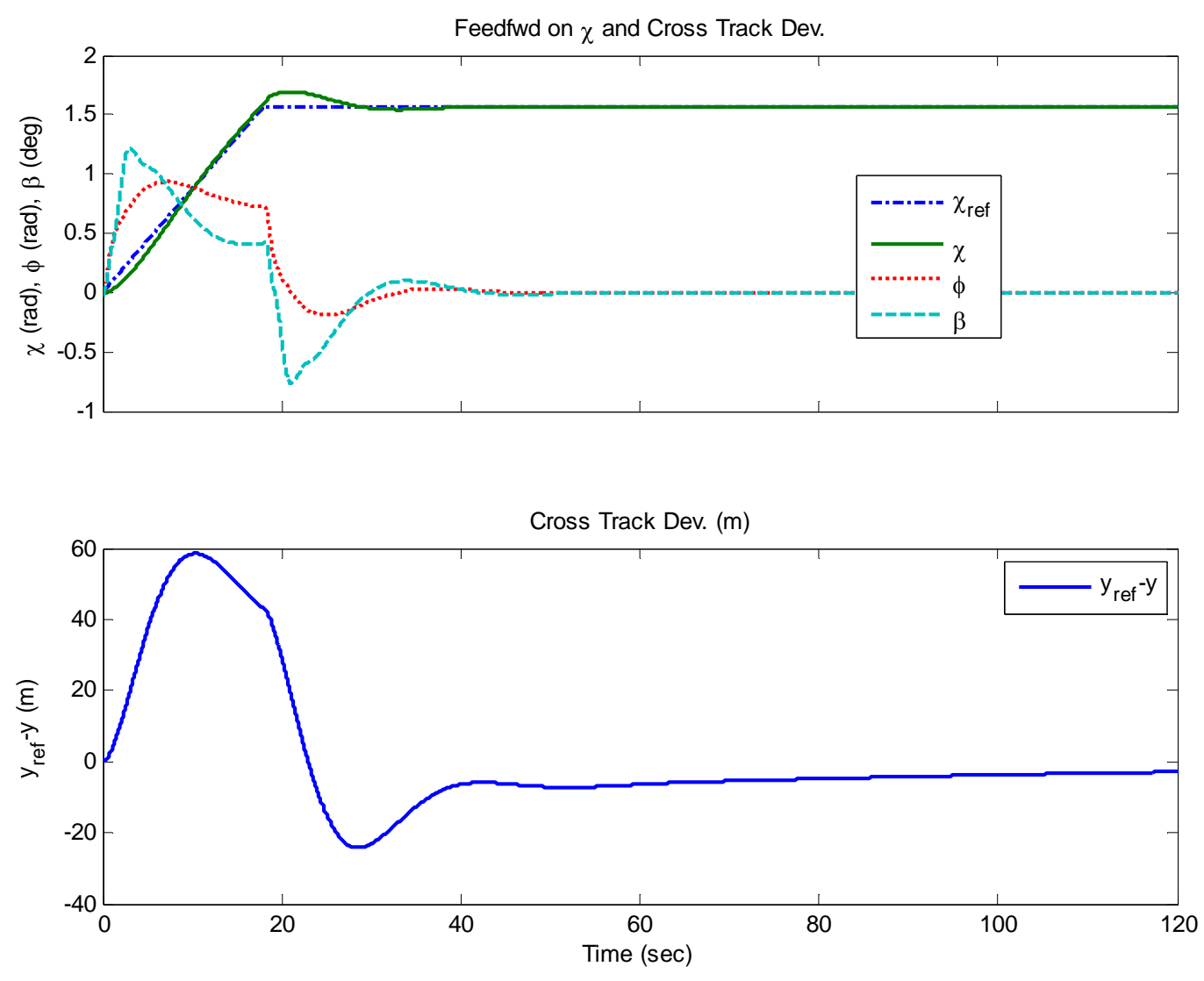

Fig. 9 Closed loop linear response of the classical lateral-directional controller. 

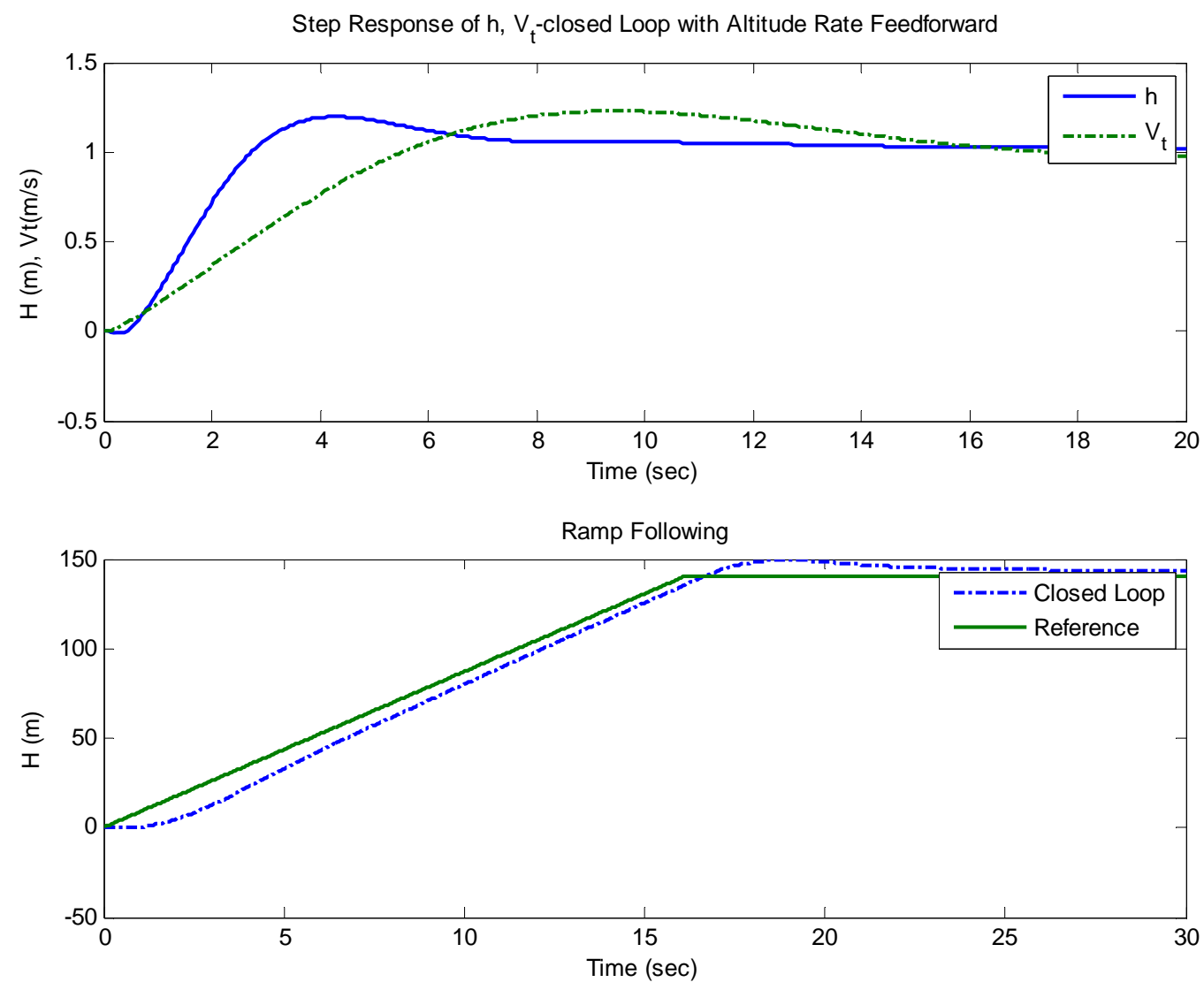

Fig. 10. Closed loop linear response of the DDBS longitudinal controller. 
Feedfwd on $\chi$ and Cross Track Dev.
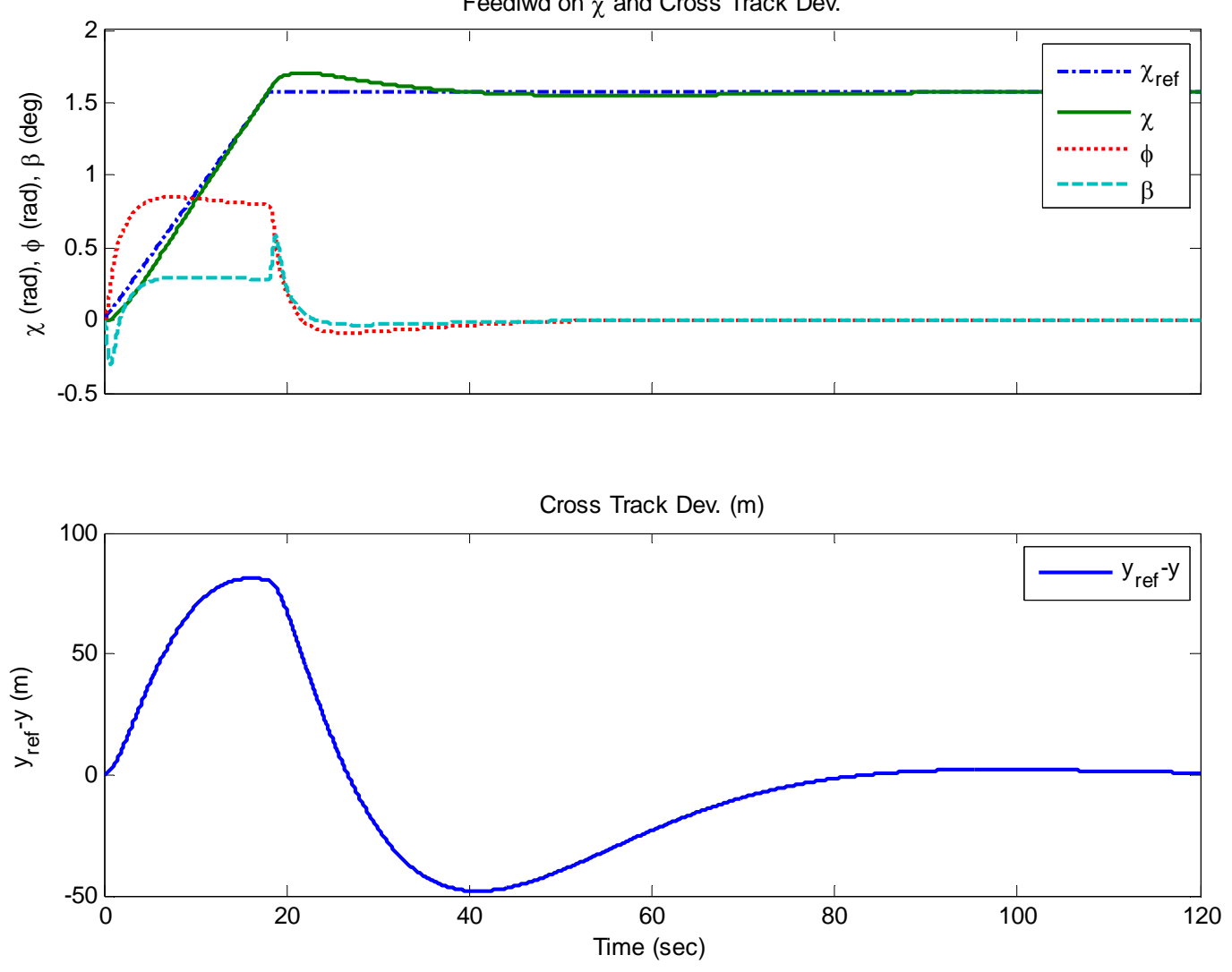

Fig. 11 Closed loop linear response of the DDBS lateral-directional controller. 

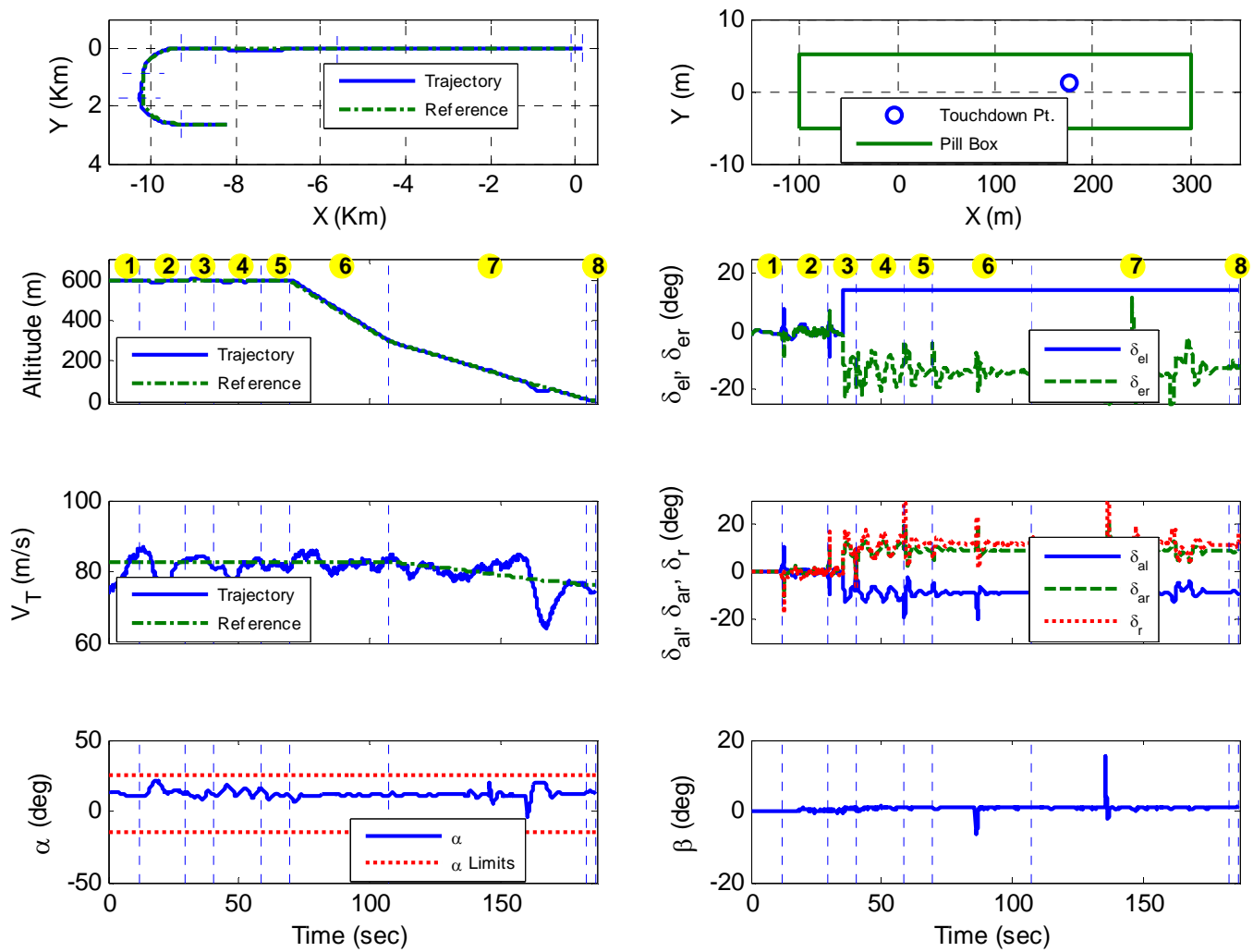

Fig. 12. Autolanding trajectories under wind disturbances and left-elevator stuck at 14 deg for the DDBS controller. 

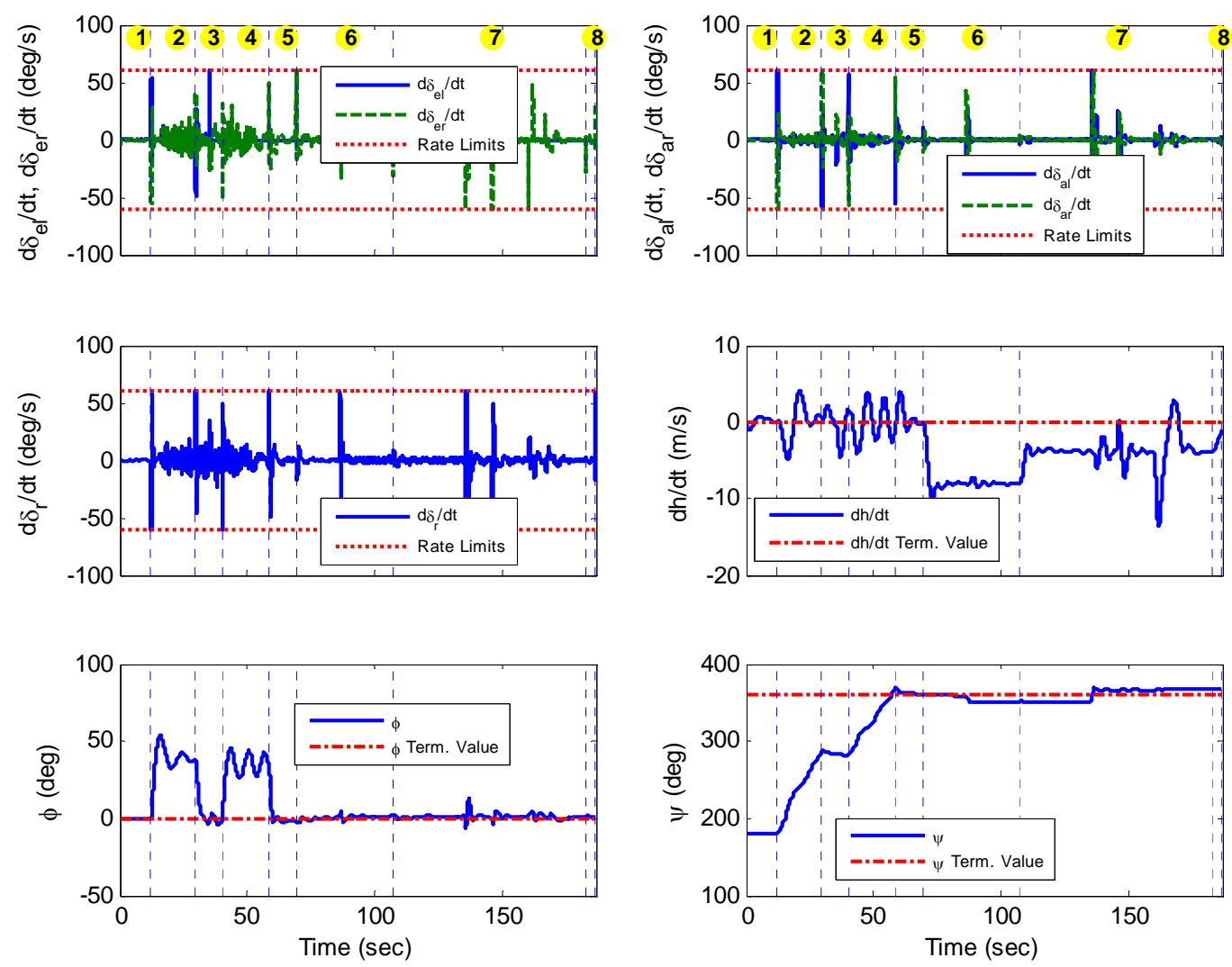

Fig. 13 Actuator Rates and State Limits under wind disturbances and left-elevator stuck at 14 deg for the DDBS controller. 

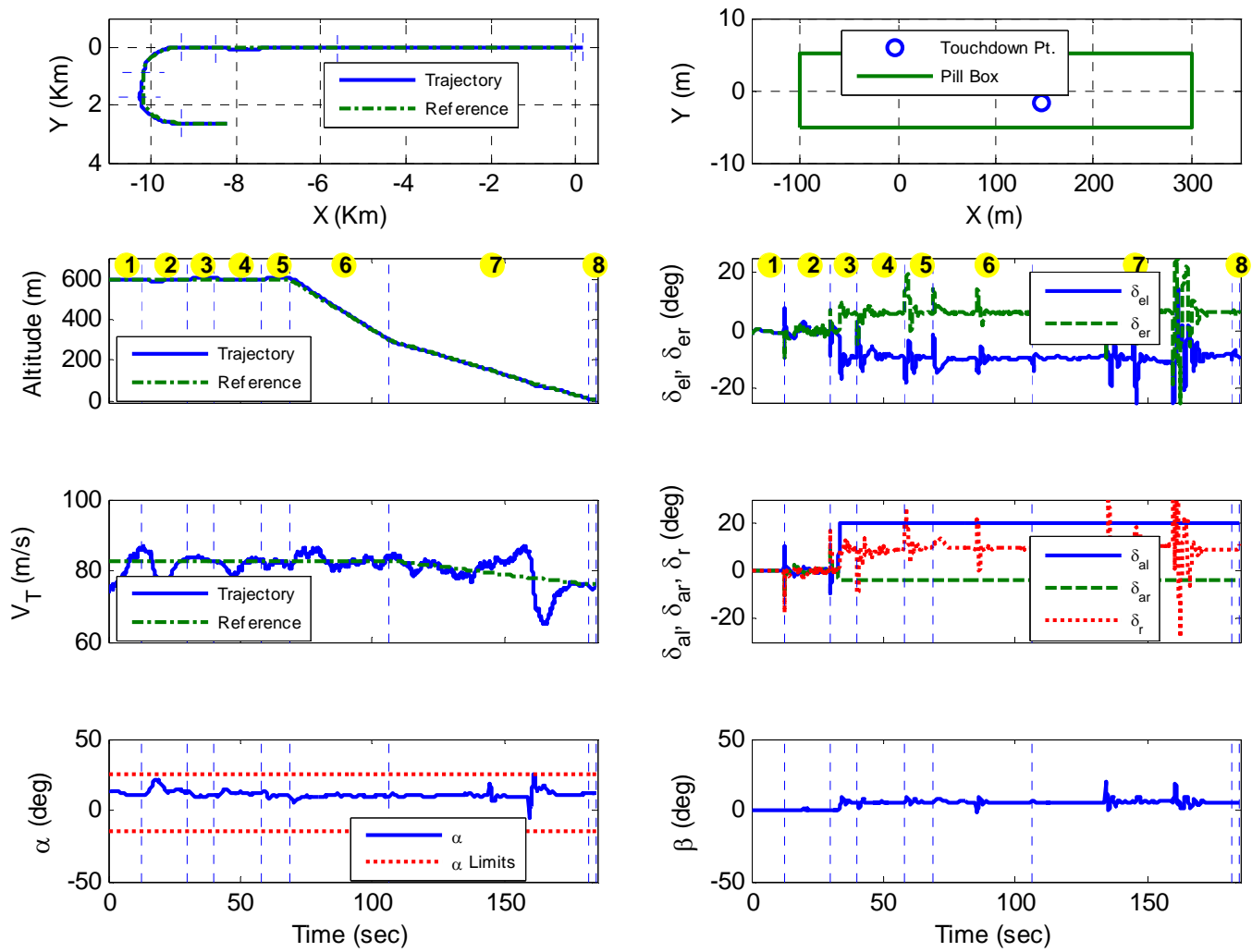

Fig. 14. Autolanding trajectories under severe wind disturbances, and left-aileron stuck at 20 deg. and right-aileron stuck at -4 deg for the DDBS controller. 

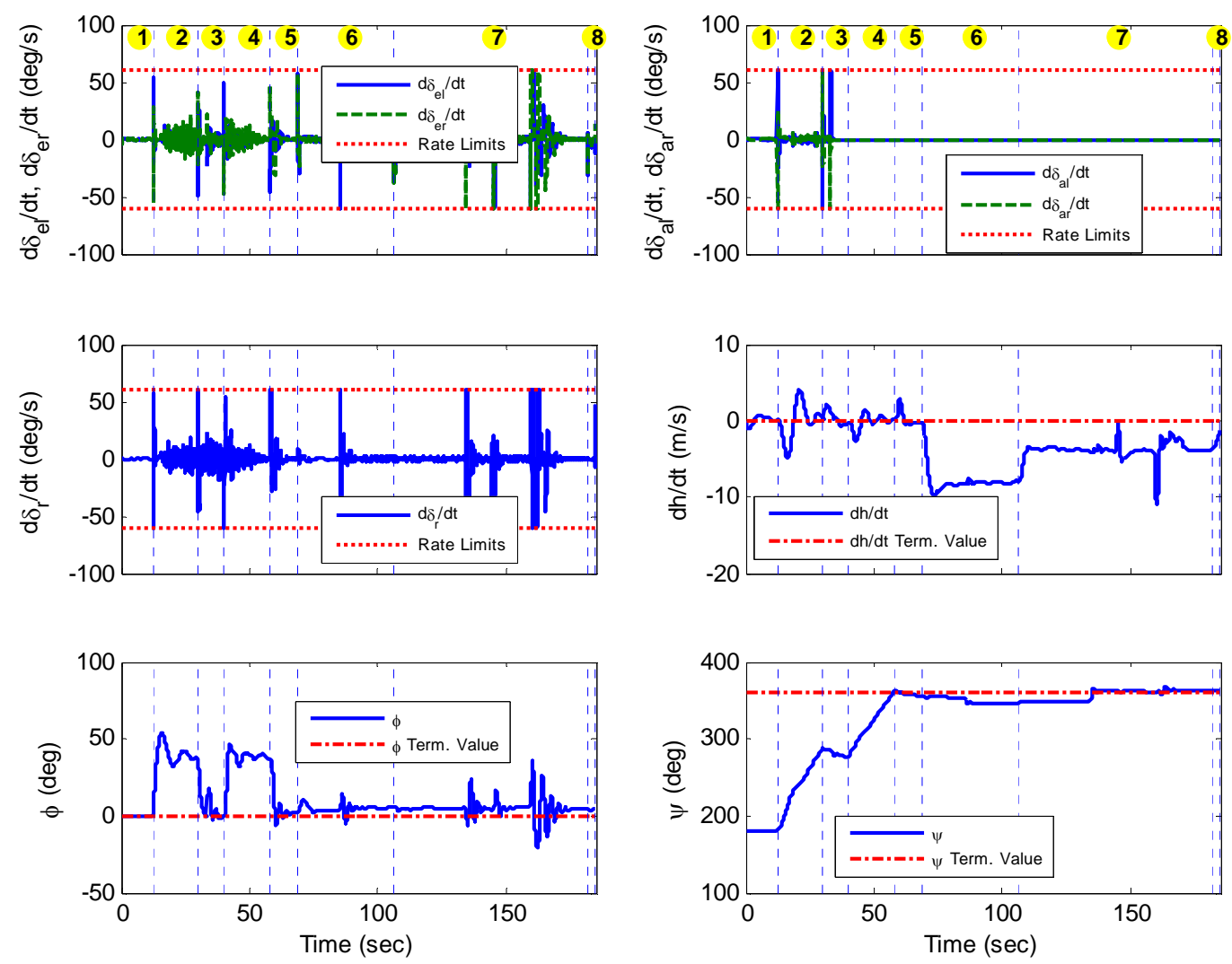

Fig. 15 Actuator Rates and State Limits severe wind disturbances, and left-aileron stuck at 20 deg. and right-aileron stuck at -4 deg for the DSBS controller. 

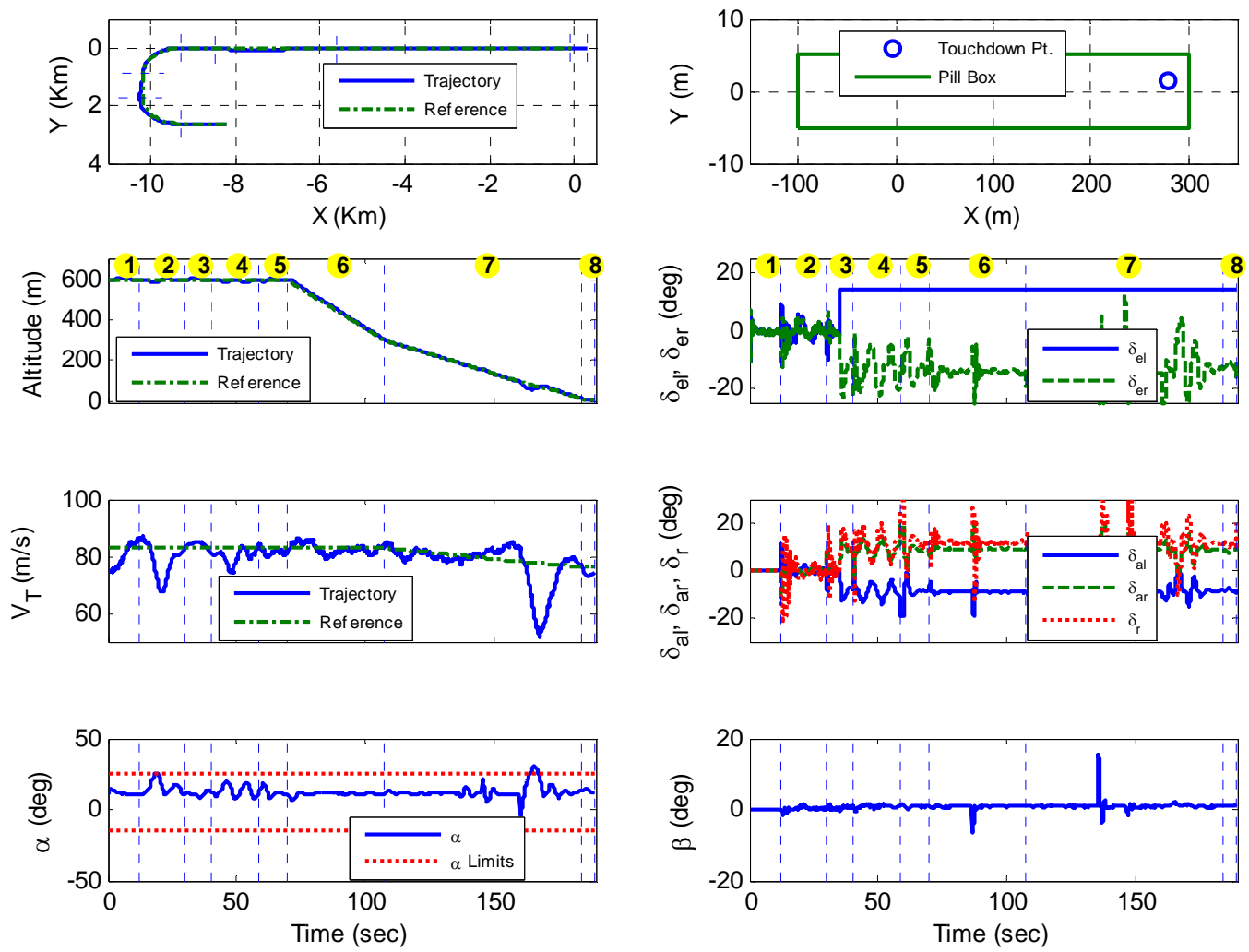

Fig. 16. Autolanding trajectories under severe wind disturbances, and left-elevator stuck at 14 deg. for the DDBS controller with angle of attack and sideslip feedback signals replaced by normal and lateral acceleration signals respectively. 

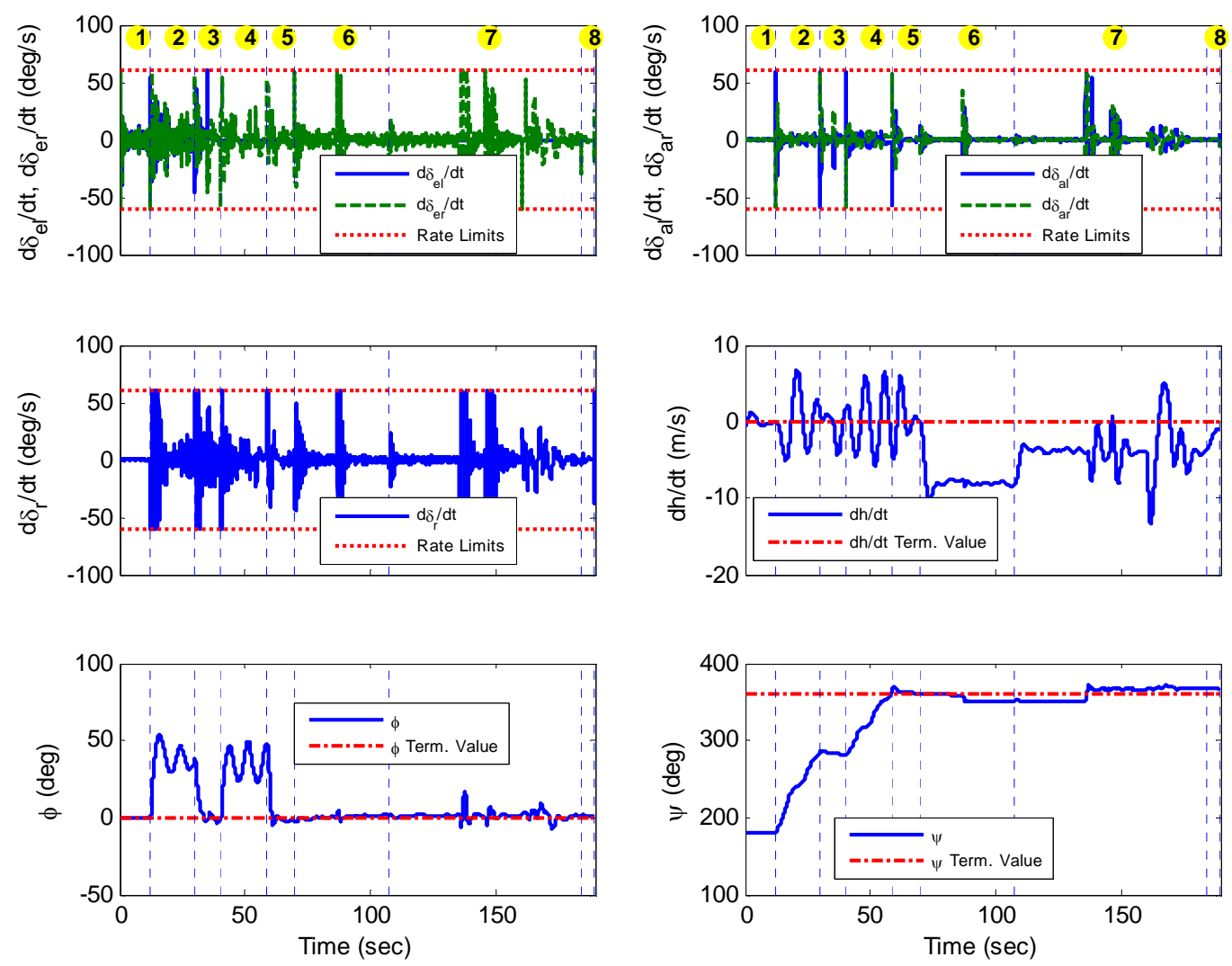

Fig. 17 Actuator Rates and State Limits severe wind disturbances, and left- elevator stuck at 14 deg. for the DDBS controller with angle of attack and sideslip feedback signals replaced by normal and lateral acceleration signals respectively. 